\title{
DOSIMETER DESIGN PROGRAM
}

Craig J. Kief

COSMIAC at UNM

2350 Alamo Avenue SE, Ste 300

Albuquerque, NM 87106

5 Jan 2015

Final Report

APPROVED FOR PUBLIC RELEASE; DISTRIBUTION IS UNLIMITED.

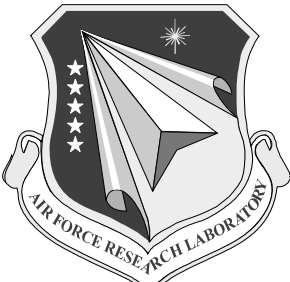

AIR FORCE RESEARCH LABORATORY

Space Vehicles Directorate

3550 Aberdeen Ave SE

AIR FORCE MATERIEL COMMAND

KIRTLAND AIR FORCE BASE, NM 87117-5776 


\section{DTIC COPY \\ NOTICE AND SIGNATURE PAGE}

Using Government drawings, specifications, or other data included in this document for any purpose other than Government procurement does not in any way obligate the U.S. Government. The fact that the Government formulated or supplied the drawings, specifications, or other data does not license the holder or any other person or corporation; or convey any rights or permission to manufacture, use, or sell any patented invention that may relate to them.

This report is the result of contracted fundamental research deemed exempt from public affairs security and policy review in accordance with SAF/AQR memorandum dated 10 Dec 08 and AFRL/CA policy clarification memorandum dated 16 Jan 09. This report is available to the general public, including foreign nationals. Copies may be obtained from the Defense Technical Information Center (DTIC) (http://www.dtic.mil).

AFRL-RV-PS-TR-2014-0168 HAS BEEN REVIEWED AND IS APPROVED FOR PUBLICATION IN ACCORDANCE WITH ASSIGNED DISTRIBUTION STATEMENT.

//SIGNED//

KEITH AVERY

Program Manager
//SIGNED//

JAMES LYKE

Tech Advisor, Space Electronics Protection Branch

//SIGNED//

BENJAMIN M. COOK, Lt Col, USAF

Deputy Chief, Spacecraft Technology Division

Space Vehicles Directorate

This report is published in the interest of scientific and technical information exchange, and its publication does not constitute the Government's approval or disapproval of its ideas or findings. 


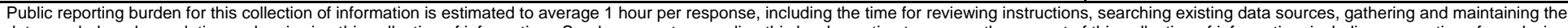

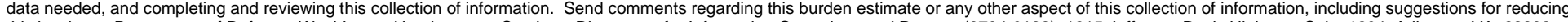

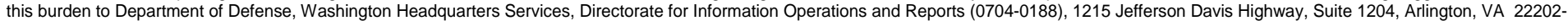

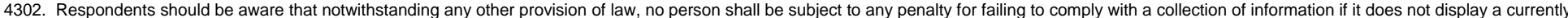
valid OMB control number. PLEASE DO NOT RETURN YOUR FORM TO THE ABOVE ADDRESS.
1. REPORT DATE (DD-MM-YY)
05-01-2015
2. REPORT TYPE
Final Report

4. TITLE AND SUBTITLE

Dosimeter Design Program

3. DATES COVERED (From - To)

16 Jul 2013 to 16 Oct 2014

5a. CONTRACT NUMBER

FA9453-13-1-0283

5b. GRANT NUMBER

5c. PROGRAM ELEMENT NUMBER

63401F

5d. PROJECT NUMBER

2181

5e. TASK NUMBER

PPM00017001

5f. WORK UNIT NUMBER

EF011499

8. PERFORMING ORGANIZATION REPORT NUMBER

COSMIAC at UNM

2350 Alamo Avenue SE, Ste 300

Albuquerque, NM 87106

\section{SPONSORING I MONITORING AGENCY NAME(S) AND ADDRESS(ES)}

Air Force Research Laboratory

Space Vehicles Directorate

3550 Aberdeen Ave., SE

Kirtland AFB, NM 87117-5776

\section{DISTRIBUTION I AVAILABILITY STATEMENT}

Approved for Public Release; Distribution is Unlimited.

\section{SPONSOR/MONITOR'S ACRONYM(S) AFRL/RVSE}

11. SPONSOR/MONITOR'S REPORT NUMBER(S)

AFRL-RV-PS-TR-2014-0168

\section{SUPPLEMENTARY NOTES}

\section{ABSTRACT}

The purpose of this research project was to explore the various aspects of dosimeter development with a particular focus on nanosatellites. The team at the University of New Mexico's COSMIAC Center created a series of two different dosimeters for space flight. The first dosimeter was for low earth orbit and as such, didn't require radiation hardened electronics. The second dosimeter was created to fly in a much higher environment so it was designed to operate with radiation hardened electronics. The developed dosimeter system is called the Radiation Hazard Assessment System (RHAS). The RHAS was developed to monitor the radiation environment in a geosynchronous satellite.

\section{SUBJECT TERMS}

Radiation testing, Cobalt, Microcontroller

\begin{tabular}{|c|c|c|c|c|c|}
\hline 16. SECURITY & IFICATION OF: & & 17. LIMITATION & 18. NUMBER & 19a. NAME OF RESPONSIBLE PERSON \\
\hline $\begin{array}{l}\text { a. REPORT } \\
\text { Unclassified }\end{array}$ & $\begin{array}{l}\text { b. ABSTRACT } \\
\text { Unclassified }\end{array}$ & $\begin{array}{l}\text { c. THIS PAGE } \\
\text { Unclassified }\end{array}$ & Unlimited & 78 & $\begin{array}{l}\text { 19b. TELEPHONE NUMBER (include area } \\
\text { code) }\end{array}$ \\
\hline
\end{tabular}


(This page intentionally left blank) 


\section{ACKNOWLEDGEMENT}

This material is based on research sponsored by Air Force Research Laboratory or the U.S. Government under agreement number FA9453-13-1-0283. The U.S. Government is authorized to reproduce and distribute reprints for Governmental purposes notwithstanding any copyright notation thereon."

\section{DISCLAIMER}

The views and conclusions contained herein are those of the authors and should not be interpreted as necessarily representing the official policies or endorsements, either expressed or implied, of Air Force Research Laboratory or the U.S. Government. 


\section{Table of Contents}

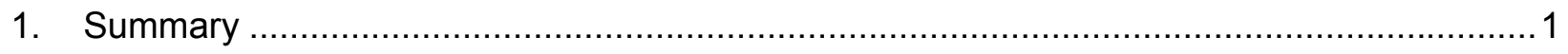

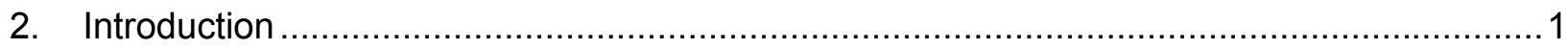

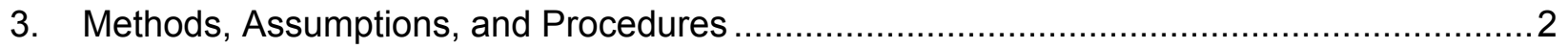

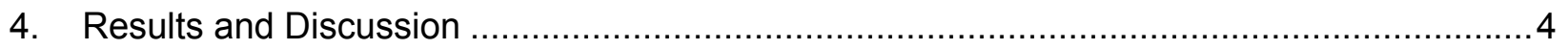

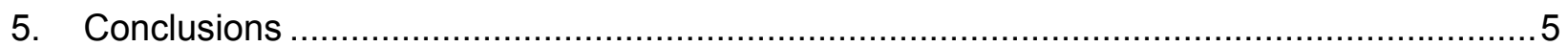

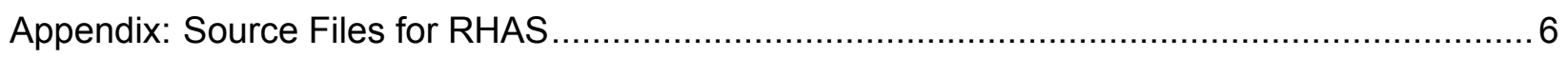

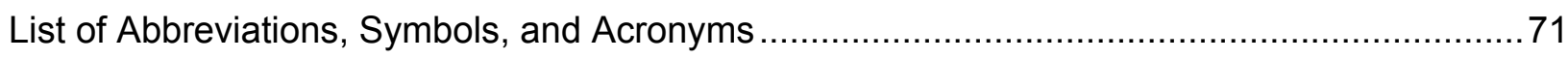

Approved for Public Release; Distribution is unlimited 


\section{Summary}

The University of New Mexico (UNM) proposed to develop a series of dosimeter flight articles for use in future small satellite missions. The Configurable Space Microsystems Innovations and Applications Center (COSMIAC) aerospace and defense center at UNM worked on two CubeSats for delivery to the National Aeronautics and Space Administration (NASA) in 2013 and 2014. Dosimeters provide a critical way to measure the radiation at various altitudes and orbital inclinations. By understanding the actual levels, this allows developers of large (and very expensive) satellites to be able to use less expensive parts that meet minimum requirements for survivability.

\section{Introduction}

The current trend in nanosatellites is the use of a standard called the CubeSat. The CubeSat form factor is described with the use of " $U$ " for units. A $1 \mathrm{U}$ is $10 \mathrm{~cm} \times 10 \mathrm{~cm} \times$ $10 \mathrm{~cm}$. A $6 \mathrm{U}$ is $10 \mathrm{~cm} \times 20 \mathrm{~cm} \times 30 \mathrm{~cm}$. UNM completed the design build and launch of a $1 U$ CubeSat called Trailblazer (see Figure 1) that was launched in November of 2013 upon the ORS-3 mission. In addition, COSMIAC is working to complete a $6 \mathrm{U}$ spacecraft called ORS Squared for launch in late 2014. This spacecraft is shown in Figure 2.

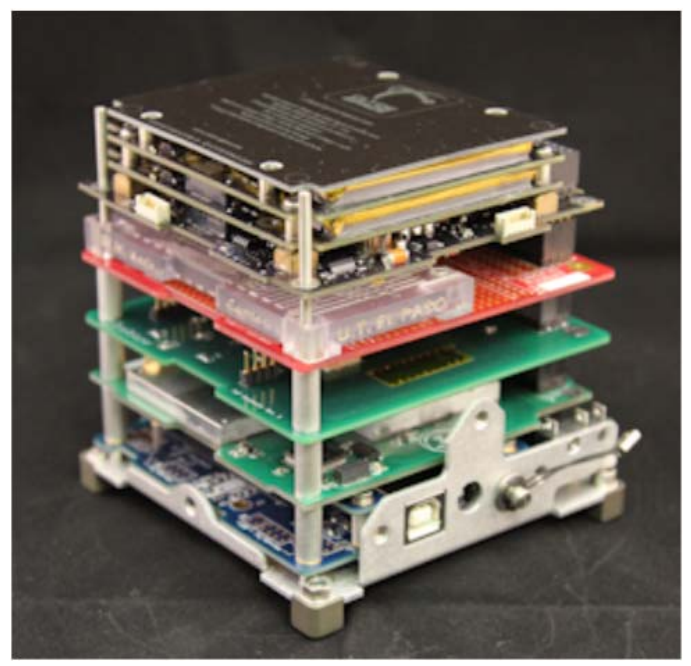

Figure 1. Trailblazer

The team said that they would use the provided funds to complete three major goals:

- Design of a dosimeter that is not radiation hardened. The team will work to create a dosimeter design using parts that are not radiation hardened. This will allow for a dosimeter that can be used on any inexpensive CubeSat designed for 
Low Earth Orbit (LEO). Satellites flown in LEO are often provided with natural protection from harmful effects normally found in the outer environment. The Earth's natural barriers provide protection for the planets occupants as well as spacecraft that stay close to the Earth's surface $(200 \mathrm{~km}-400 \mathrm{~km})$ from many of the effects of solar radiation. This means that standard commercial electronics (which are much cheaper) can often be utilized. Non-hardened parts can often be an order of magnitude less in cost.

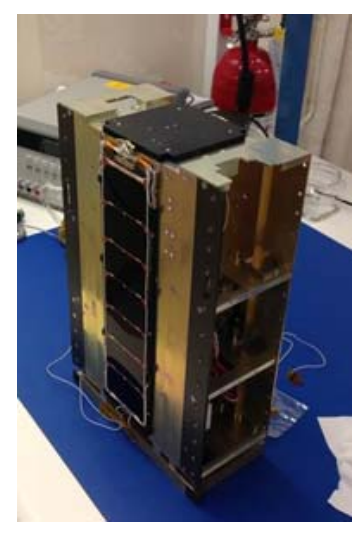

Figure 2. ORS

- Design a dosimeter that is radiation hardened. We will use the funding to create initial designs for a dosimeter that is built on radiation hardened parts designed for much higher orbits. There will be no prototype developed under this activity since the cost for the radiation hardened parts would be prohibitive. Various chips that are radiation hardened will be purchased for analysis.

- Develop Space Plug-and-play Architecture (SPA) interfaces for the dosimeters. The Air Force Research Laboratory has created a bus architecture call the SPA. This design paradigm allows for easy integration of modules into a spacecraft or other system. The team will work to develop a pathway ahead to make sure that both versions of the dosimeter could have a SPA interface.

\section{Methods, Assumptions, and Procedures}

Goal 1: The team created a simple non hardened dosimeter that was completed in time to be flown on the Trailblazer satellite in November of 2013. Unfortunately, this satellite was never heard from once on orbit so minimum results from on orbit are available. However, this design was also evaluated for flight in future COSMIAC satellites. An example schematic for the board is shown in Figure 3 (full schematics are provided upon request). We believe there is significant value to this design in its simplicity. We began work with other schools within the AFRL University Nanosat Program (UNP) to see if this is a sensor that they would like to fly on their spacecraft. Its design (with its low power and inexpensive parts) makes it an excellent sensor platform for a UNP satellite. We can provide any UNP partner with the complete schematics, bill of materials and board design files. 


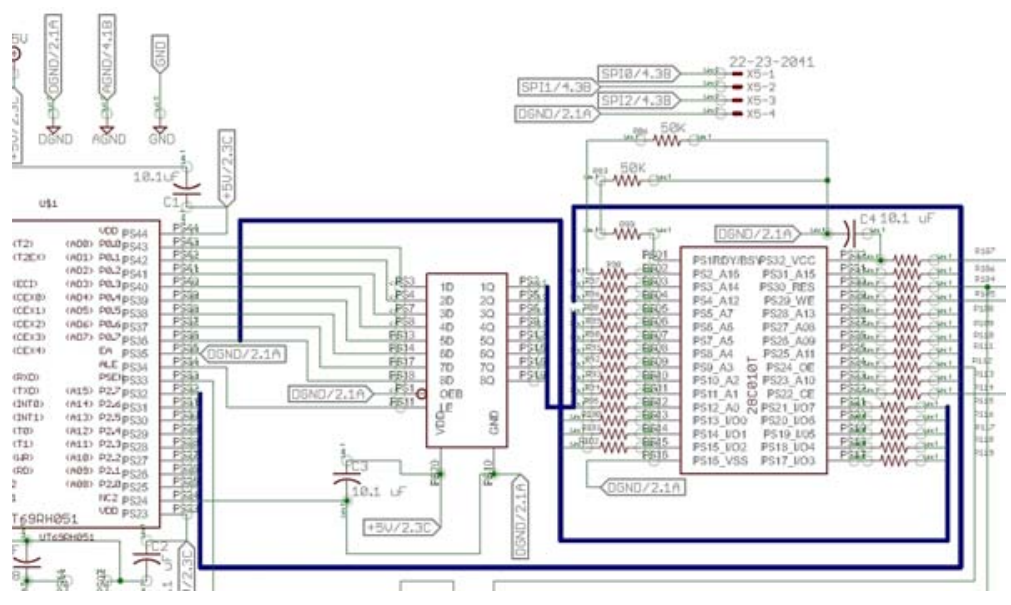

Figure 3. Board schematic subset

Goal 2: The radiation hardened version of this dosimeter board has been designed. Although there was no initial intent to actually create the hardware platform, resources and unforeseen opportunities provided the ability for the team to actually create this type of platform. We created the board as shown in Figure 4. COSMIAC has coordinated to find a flight opportunity for this dosimeter board on a Massachusetts Institute of Technology (MIT)/Lincoln Lab satellite for 2015. Engineering models were created and provided to integration teams at MIT. All problems that have been identified so far have been resolved. The schematics for the board are very similar to the ones shown in Figure 3 for the non-hardened version. The main difference is the use of parts that can withstand the rigors of deep space. With the assistance of AFRL's Space Weather Center of Excellence, we were able to do flight environmental testing and qualification. Electromagnetic interference testing identified potential areas of concern that were addressed by the inclusion of increased metal sheeting. AFRL Space Vehicles personnel assisted with total dose radiation testing on Kirtland Air Force Base. The developed dosimeter system was called the Radiation Hazard Assessment System (RHAS). The RHAS was originally developed to monitor the radiation environment in a geosynchronous satellite. It incorporates three Teledyne $\mu \mathrm{Dos} 001$ dosimeters to measure total ionizing dose resulting from either proton or electron strikes in the space environment. The dosimeters employ a silicon detector which responds to energy deposits in the range of 100 kilo-electron volt to 15 Mega-electron volt. The current pulses from the detector are integrated, and when the resulting charge quanta reach a threshold equivalent to 14 micro-radians, a counter is incremented. The counter output is applied to a digital to analog converter which drives the three output ranges (low, medium, and high) that are progressively scaled by 256 (i.e., medium range $=256^{\star}$ low range, etc. . 
The RHAS instrument shown in Figure 4 was designed to fit in a CubeSat format. The three dosimeters are located on the top side of the instrument board. In their current application, a different effective thickness of shielding (not shown here) is applied over each dosimeter to provide an indication of the energy spectrum of the space radiation. The instrument incorporates radiation hardened electronics including: an Aeroflex 8051 microcontroller, a Maxwell Electrically Erasable Programmable Read-Only Memory (EEPROM), Texas Instrument analog to digital converters, and associated support electronics. Appendix A contains the source code used for this instrument. The electronics are specified to a hardness level of 1 Mega-radian/Silicon total dose and are latch-up free. COSMIAC can provide additional radiation testing to higher total dose levels if required.

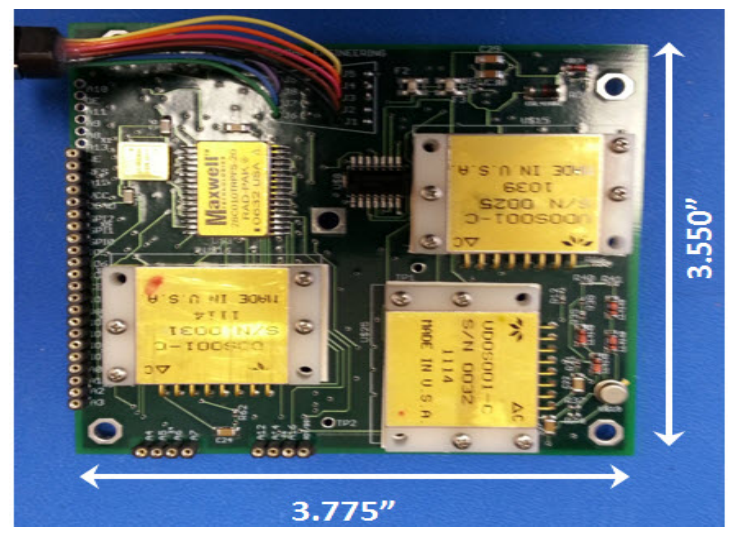

Figure 4. Flight model of RadHard Dosimeter

Goal 3: The tricky part of SPA is that the versions changed in the middle of the project. The original version that existed when this proposal was written was called the Satellite Data Module (SDM). The new version is called the Satellite System Module (SSM). SSM is much more mature and of higher quality. Also, this software has now been released as open source by AFRL. This greatly increases the ability to utilize it for student projects. The non-hardened version of the dosimeter board is already SPA compatible (older SDM version). The development of the SPA version of the hardened board was evaluated and then decided against. Future plans for SPA within the larger satellite community are very much in a state of flux so it was deemed that doing this development (at this time) would provide very limited benefit. Evaluation was made on what needed to be accomplished should this be desired later. Hardware and software stubs have been put into place to allow for easy integration at a later time.

\section{Results and Discussion}

The results to date are that the first iterations of the boards have been created. Ongoing testing has begun but will need to be continued for months to completely confirm that the systems are operating within acceptable parameters. Since a launch opportunity has been found, the next iterations will involve the creation of a control system to be able to interface the dosimeter into a larger orbital platform. 


\section{Conclusions}

The conclusions are that it is possible for an academic institution to be able to create a radiation detection system on a limited budget that has space community level utility to achieve the required work for measurements in a space environment.

Approved for Public Release; Distribution is unlimited 


\section{Appendix: Source Files for RHAS}

\section{File List}

Here is a list of all files with brief descriptions:

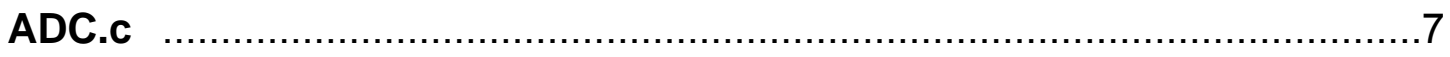

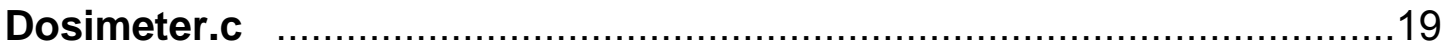

Interface.c …

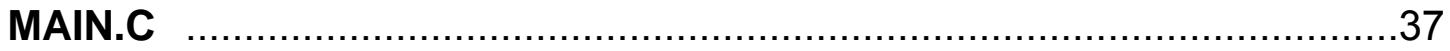

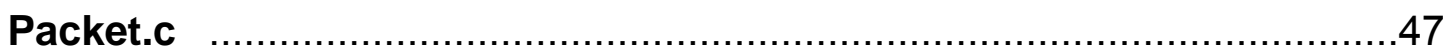

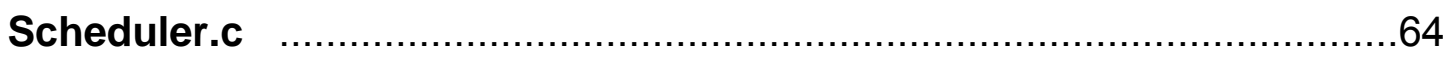

SIO.C

Approved for Public Release; Distribution is unlimited 
File Documentation

ADC.c File Reference

\#include "main.h"

Functions

- tByte read_dosimeter_all (tByte mode2)

- tByte read_dosimeter_low ()

- tByte check_rollover (tByte val, tByte old_val)

- tWord compute_avg (tWord x, tWord xmean)

Variables

- tByte tick $=0$

- tByte mode

- tByte count $=0$

- tWord curr_val

- tByte $\mathbf{k}[]=\{0,0,0,0,0\}$

- tLong idata dos [3] INSTRUMENT CONTROL Dosimeter Telemetry Application (Main module) (Version 2.0: Flight Software)

- tLong idata dos_zero [3]

- tLong idata cal_pre [3]

- tLong idata cal_post [3]

- tLong idata TickCount

- Status1_type idata Status1

- tByte code adc_ch [20]

- tByte code adc_ch_mode2 [3][5]

- tWord idata ps_15V

- tWord idata ps_5V

- tWord idata temps [3]

- bit RunOnce

- bit ReadAlllsRunning

\section{Function Documentation}

tByte check_rollover (tByte val, tByte old_val)

Definition at line 325 of file ADC.c.

tWord compute_avg (tWord $x$, tWord xmean) 
Definition at line 338 of file ADC.C.

tByte read_dosimeter_all (tByte mode2)

Definition at line 45 of file ADC.c.

tByte read_dosimeter_low ()

Definition at line 227 of file ADC.c.

\section{Variable Documentation}

tByte code adc_ch[20]

Definition at line 104 of file MAIN.C.

tByte code adc_ch_mode2[3][5]

Definition at line 91 of file MAIN.C.

tLong idata cal_post[3]

Definition at line 36 of file MAIN.C.

tLong idata cal_pre[3]

Definition at line 35 of file MAIN.C.

tByte count $=0$

Definition at line 8 of file ADC.c.

tWord curr_val

Definition at line 9 of file ADC.c.

tLong idata dos[3]

INSTRUMENT CONTROL Dosimeter Telemetry Application (Main module) (Version 2.0: Flight Software)

This code implements the full INSTRUMENT CONTROL dosimeter sensor, including all message formatting, data processing, and utility functions (ADC, CRC, ...)

Approved for Public Release; Distribution is unlimited 
Version Rev. 1.0 Created from the code written by Brian Zufelt for the development board and by code written by me for the EU model.

Chip type : Aeroflex UTMC UT69RH051 Program type : Firmware Core Clock frequency : $16.129000 \mathrm{MHz}$

Definition at line 33 of file MAIN.C.

tLong idata dos_zero[3]

Definition at line 34 of file MAIN.C.

tByte $k \square=\{0,0,0,0,0\}$

Definition at line 10 of file ADC.C.

tByte mode

Definition at line 7 of file ADC.c.

tWord idata ps_15V

Definition at line 38 of file MAIN.C.

tWord idata ps_5V

Definition at line 39 of file MAIN.C.

bit ReadAlllsRunning

Definition at line 81 of file MAIN.C.

bit RunOnce

Definition at line 84 of file MAIN.C.

Status1_type idata Status1

Definition at line 78 of file MAIN.C.

tWord idata temps[3]

Definition at line 40 of file MAIN.C.

Approved for Public Release; Distribution is unlimited 
tByte tick $=0$

Definition at line 6 of file ADC.c.

tLong idata TickCount

Definition at line 57 of file MAIN.C.

Approved for Public Release; Distribution is unlimited 
// ADC.C

// This code implements all variants of the ADC functions

\#include "main.h"

tByte tick = 0;

tByte mode;

tByte count $=0$;

tWord curr_val;

tByte $k[]=\{0,0,0,0,0\}$;

extern tLong idata dos[3];

extern tLong idata dos_zero[3];

extern tLong idata cal_pre[3];

extern tLong idata cal_post[3];

extern tLong idata TickCount;

extern Status1_type idata Status1;

extern tByte code adc_ch[20];

extern tByte code adc_ch_mode2[3][5];

extern tWord idata ps_15V;

extern tWord idata ps_5V;

extern tWord idata temps[3];

extern bit RunOnce;

extern bit ReadAllIsRunning;

* read_dosimeter_all()

* Reads the actual values of the high, medium, and low channels

* of a dosimeter and stores the results in a 32-bit value (in the

* form: High:12, Med:12, Low:8

*

* This is done anytime we want the actual values (rather than the

* accumulated dose over a 2-minute period).

*

* Inputs:

* Formal name Type Purpose

*

* Return value:

* Type Purpose

* tByte $0=$ Success

* $\quad-1=$ Error: NULL pointer

* $\quad-2=$ Error: Invalid Dosimeter number

$* * * * * * * * * * * * * * * * * * * * * * * * * * * * * * * * * * * * * * * * * * * * * * * * * * * * * * * * * * * * * * * * * * * * * * * /$

tByte read_dosimeter_all( tByte mode2 )

Approved for Public Release; Distribution is unlimited 


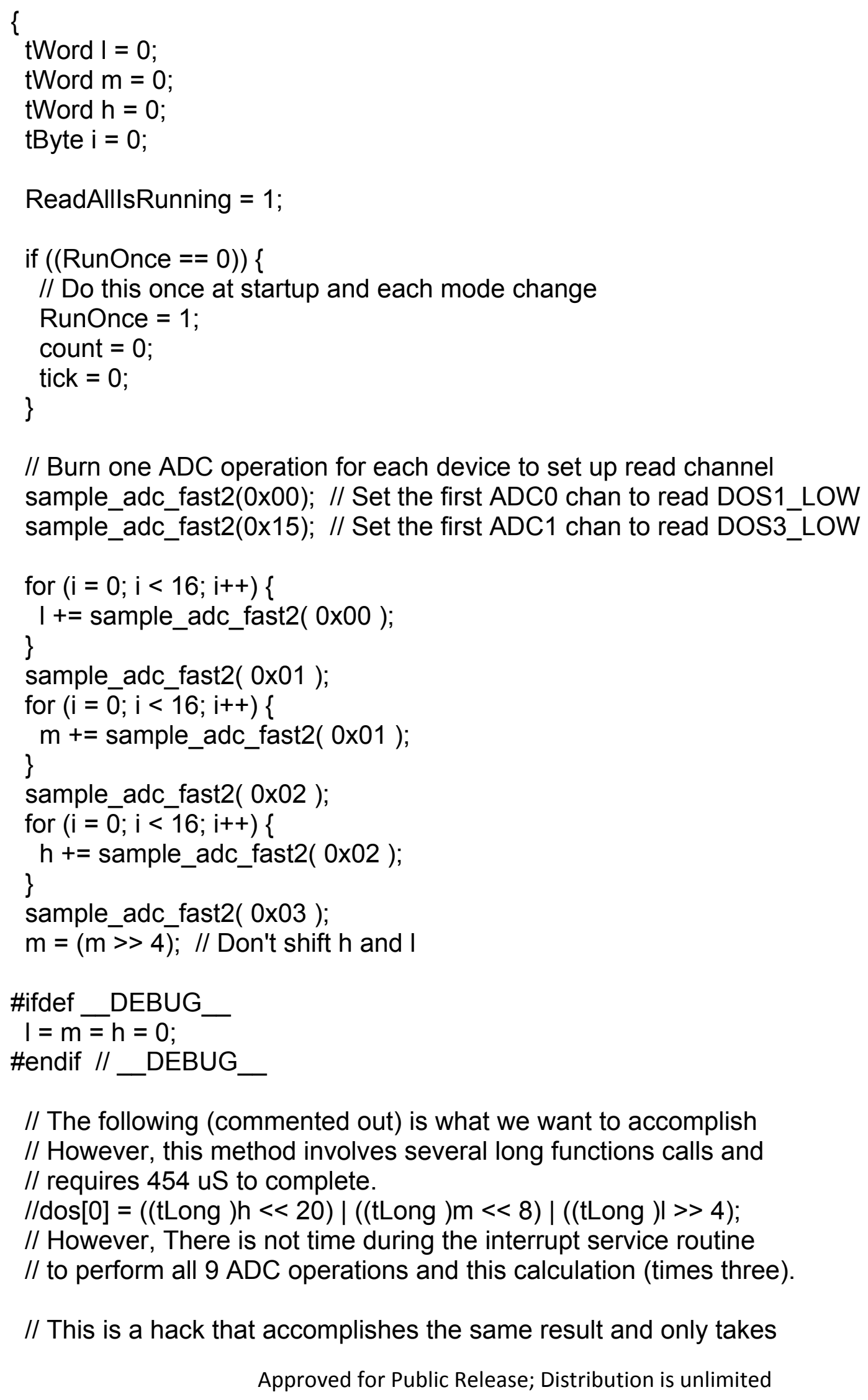

// Burn one ADC operation for each device to set up read channel sample_adc_fast2(0x00); // Set the first ADC0 chan to read DOS1_LOW sample_adc_fast2(0x15); // Set the first ADC1 chan to read DOS3_LOW

// The following (commented out) is what we want to accomplish // However, this method involves several long functions calls and // requires 454 uS to complete. //dos[0] = ((tLong $) \mathrm{h}<<20) \mid(($ tLong $) \mathrm{m}<<8) \mid(($ tLong $) \mid>>4)$; // However, There is not time during the interrupt service routine // to perform all 9 ADC operations and this calculation (times three).

// This is a hack that accomplishes the same result and only takes

Approved for Public Release; Distribution is unlimited 
// 30.5 uS to complete!! This is the magic of hand coding.

// The next two lines are commented out because they are offset by

// the collection of 16 samples.

$/ / \mathrm{h}=\mathrm{h} \ll<4$

$/ / \mathrm{I}=\mathrm{I}<<4$

${ }^{*}\left(\right.$ tByte $\left.{ }^{*}\right)($ dos $)={ }^{*}($ tByte $*)(\& h) ;$

${ }^{*}\left(\left(\right.\right.$ tByte $\left.{ }^{*}\right)$ dos +1$)=\left({ }^{*}(((\right.$ tByte $\left.*) \& \mathrm{~h})+1) \& 0 x \mathrm{xF}\right) \mid\left({ }^{*}\left(\right.\right.$ tByte $\left.\left.{ }^{*}\right)(\& \mathrm{~m}) \& 0 \mathrm{xOF}\right)$;

${ }^{*}\left(\left(\right.\right.$ tByte $\left.{ }^{*}\right)$ dos +2$)={ }^{*}(($ tByte $\left.*) \& m)+1\right)$;

${ }^{*}\left(\left(\right.\right.$ tByte $\left.{ }^{*}\right)$ dos +3$)=*\left(\right.$ tByte $\left.{ }^{*}\right)(\& \mathrm{l})$;

// Zero local values

$\mathrm{I}=\mathrm{m}=\mathrm{h}=0$;

// Read DOS2_LOW sixteen times

for $(i=0 ; i<16 ; i++)\{$

$\mathrm{I}+=$ sample_adc_fast $2(0 \times 03)$;

\}

sample_adc_fast2( $0 \times 04)$;

// Read DOS2_MID sixteen times

for $(i=0 ; i<16 ; i++)\{$

\}

$\mathrm{m}+=$ sample_adc_fast $2(0 \times 04)$;

sample_adc_fast2( 0x05);

// Read DOS2 $\mathrm{HI}$ sixteen times

for $(i=0 ; i<16$; $i++)\{$

$\mathrm{h}+=$ sample_adc_fast2(0x05);

\}

$\mathrm{m}=(\mathrm{m}>>4) ; / /$ Don't shift $\mathrm{h}$ and I

// If we are in Analysis Mode 2, we need to read subcommutated values if (mode2 == 1) \{ sample_adc_fast2(adc_ch_mode2[0][count]); // "Decision Stage 0" \} else \{ \} sample_adc_fast2( $0 \times 00)$;

\#ifdef _DEBUG

$\mathrm{h}=\mathrm{m}=\mathrm{l}=0$;

\#endif //_DEBUG

${ }^{*}\left(\right.$ tByte $\left.{ }^{*}\right)($ dos +4$)={ }^{*}\left(\right.$ tByte $\left.{ }^{*}\right)(\& h) ;$

Approved for Public Release; Distribution is unlimited 


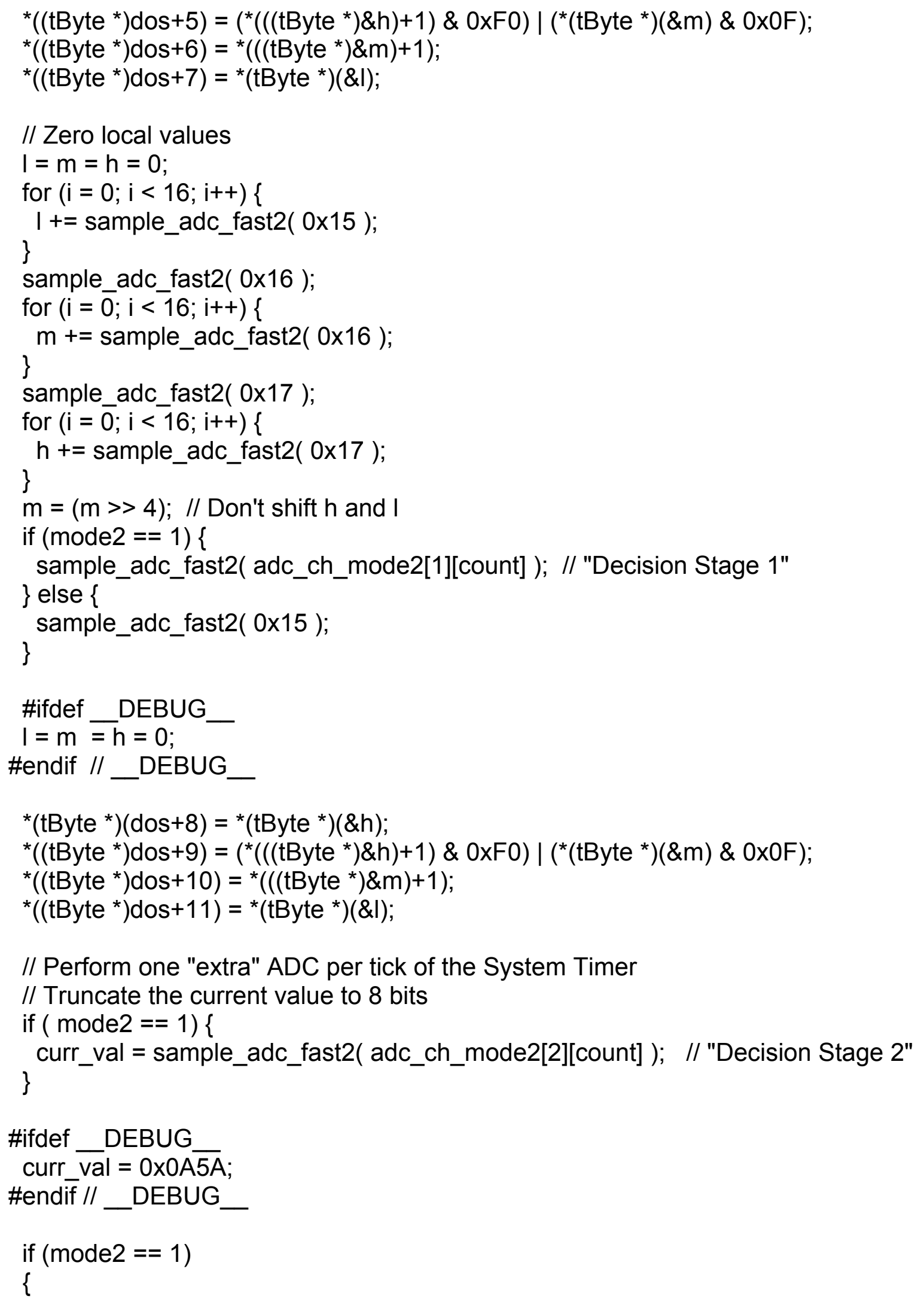

Approved for Public Release; Distribution is unlimited 


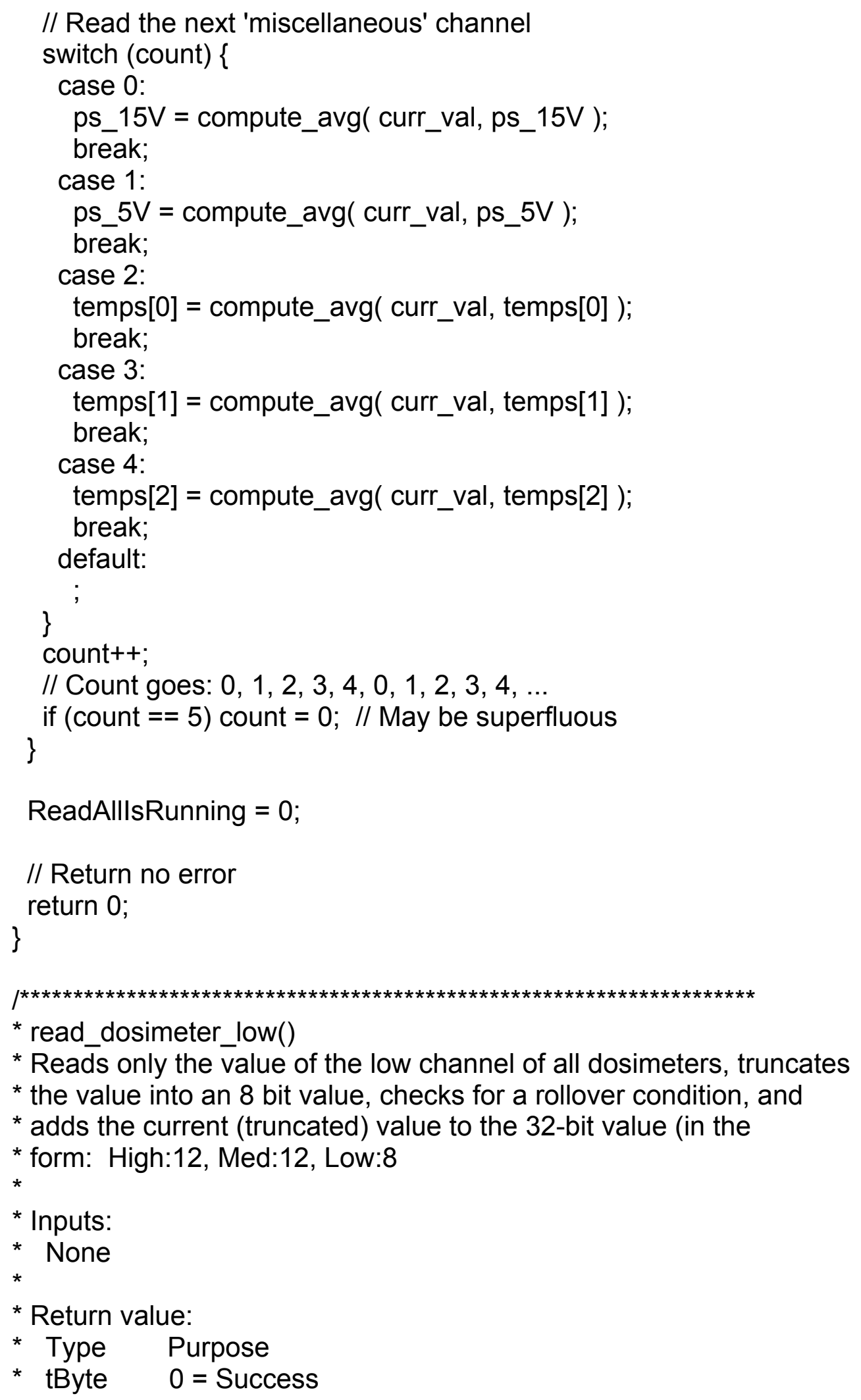




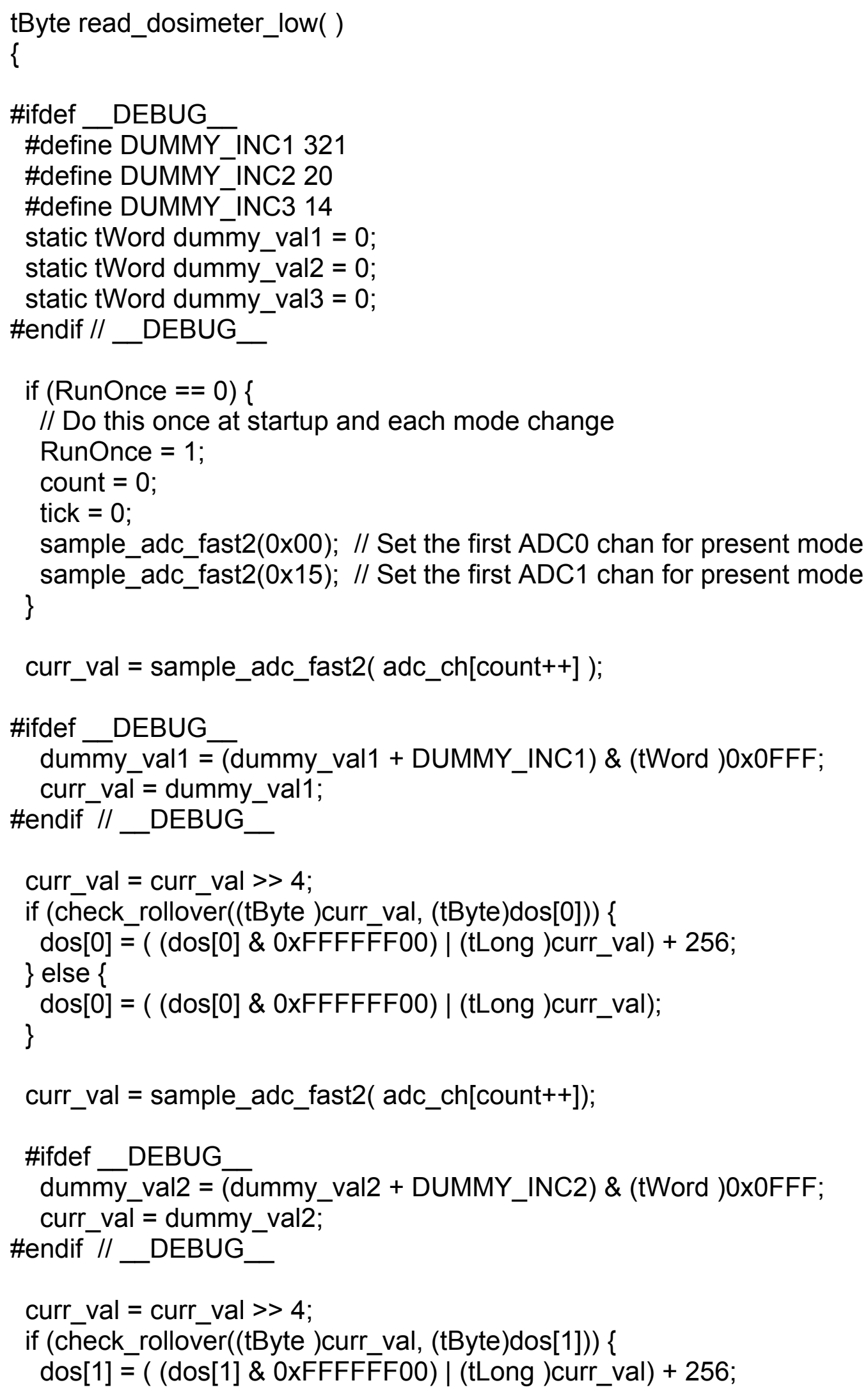




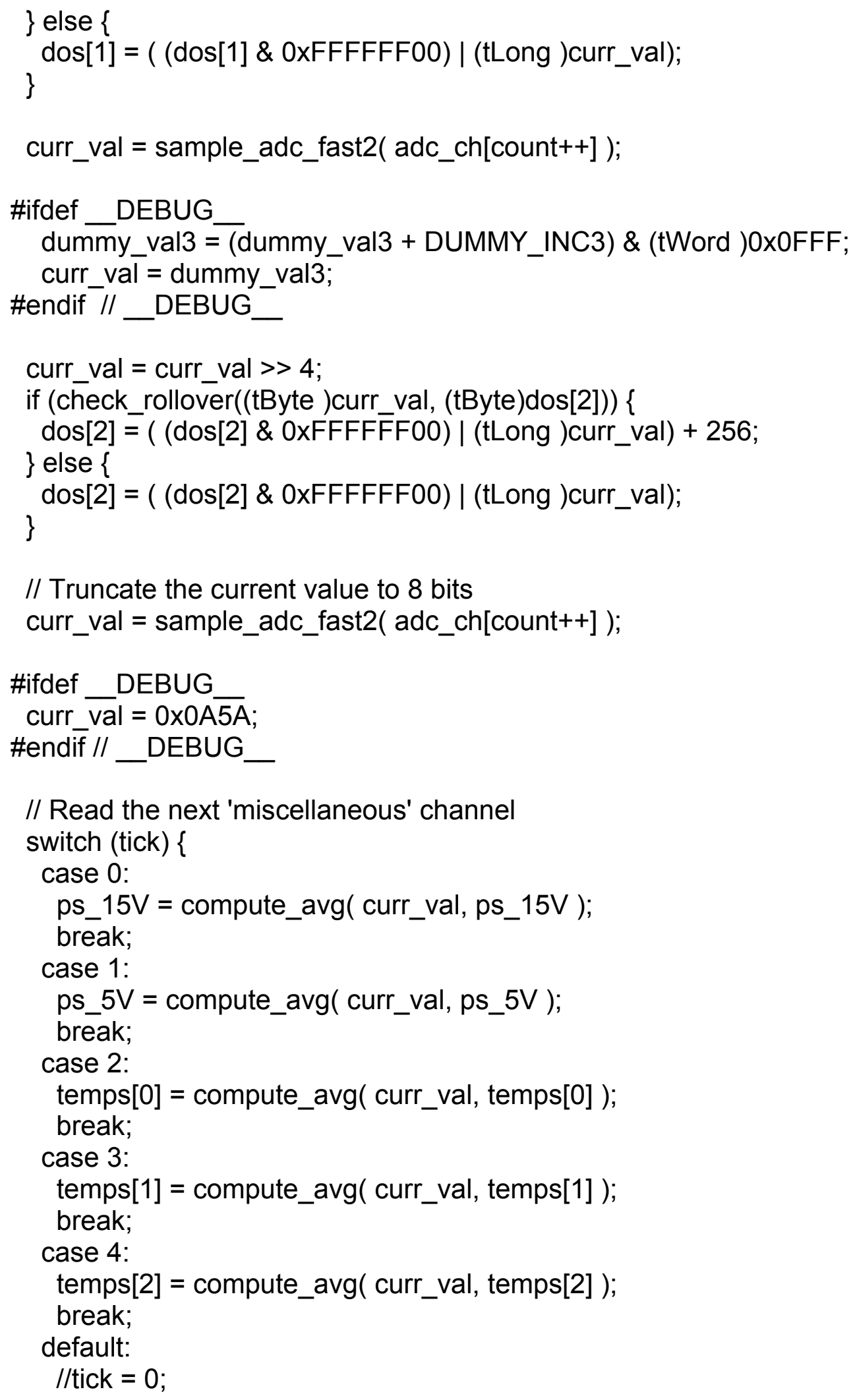




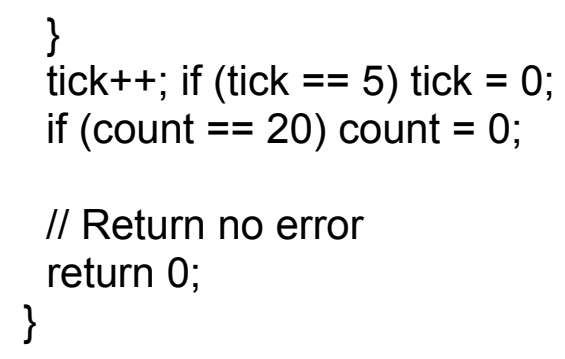

Old value: $11 x x \mathrm{xxxx}$

New value: $00 x x$ xxxx

*/

if $((($ old_val \& $0 \times C 0)==0 \times C 0) \& \&(($ val $\& 0 \times C 0)==0 \times 00))$ return 1 ; // Rollover detected

else

\}

return 0;

tWord compute_avg( tWord $\mathrm{x}$, tWord xmean )

\{

tWord xtemp;

if $(k[$ tick $]==0)\{$

$\mathrm{k}[\mathrm{tick}]=1$;

xtemp $=\mathrm{x}<<4$

\} else \{

xtemp $=$ xmean $>>1 ; / /$ xtemp $=1 / 2 *$ xmean

xtemp $+=$ xmean $>>2 ; / / x$ temp $=3 / 4{ }^{*}$ xmean

xtemp $+=x$ mean $>>3$; // xtemp $=7 / 8$ * xmean

xtemp $+=$ xmean $>>4$; // xtemp $=15 / 16 *$ xmean

$\mathrm{xtemp}+\mathrm{x} ; \quad / / \mathrm{xtemp}=\left(15 / 16{ }^{*} \mathrm{xmean}\right)+\mathrm{x}$

\}

return xtemp;

\}

Approved for Public Release; Distribution is unlimited 


\section{Dosimeter.c File Reference}

\#include "main.h"

\section{Macros}

- \#define CAL_PULSE_WIDTH 17

\section{Functions}

- void Init_INSTRUMENT CONTROL (void)

- void SysTimerSetupFast (void)

- void SysTimerSetupSlow (void)

- void SystemTick (void)

- void TSK_DoCal ()

- void initialize_normal_mode (void)

- void initialize_startup_mode (void)

- void ChangeToAnalysisMode2 (void)

- void ChangeToAnalysisMode1 (void)

- void TSK_DoADC (void)

\section{Variables}

- Status1_type idata Status1

- Status2_type idata Status2

- MCF_type idata MCF

- tLong idata dos [3]

INSTRUMENT CONTROL Dosimeter Telemetry Application (Main module) (Version

2.0: Flight Software)

- tLong idata dos_zero [3]

- tLong idata cal_pre [3]

- tLong idata cal_post [3]

- tWord idata dos_livetime [3]

- tLong idata TickCount

- tLong idata TotalTickCount

- tByte idata HourCount

- bit TelemetryTick

- bit ADC_Start

- bit CalRunning

- bit ReadAllIsRunning

- bit CalMode

- bit RunOnce

- bit GSE

- bit INSTRUMENT CONTROL_Enable

- bit Dos1TurnOn

- bit Dos2TurnOn

- bit Dos3TurnOn 
- tLong idata RxTimer

Macro Definition Documentation

\#define CAL_PULSE_WIDTH 17

Definition at line 5 of file Dosimeter.c.

Function Documentation

void ChangeToAnalysisMode1 (void)

Definition at line 279 of file Dosimeter.c.

void ChangeToAnalysisMode2 (void)

Definition at line 269 of file Dosimeter.c.

void Init_INSTRUMENT CONTROL (void)

Definition at line 31 of file Dosimeter.c.

void initialize_normal_mode (void)

Definition at line 210 of file Dosimeter.c.

void initialize_startup_mode (void)

Definition at line 239 of file Dosimeter.c. void SystemTick (void)

Definition at line 113 of file Dosimeter.c. void SysTimerSetupFast (void)

Definition at line 57 of file Dosimeter.c. void SysTimerSetupSlow (void)

Definition at line 85 of file Dosimeter.c.

Approved for Public Release; Distribution is unlimited 
void TSK_DoADC (void)

Definition at line 289 of file Dosimeter.c.

void TSK_DoCal ()

Definition at line 162 of file Dosimeter.c.

\section{Variable Documentation}

bit ADC_Start

Definition at line 47 of file MAIN.C.

tLong idata cal_post[3]

Definition at line 36 of file MAIN.C.

tLong idata cal_pre[3]

Definition at line 35 of file MAIN.C.

bit CalMode

Definition at line 54 of file MAIN.C.

bit CalRunning

Definition at line 53 of file MAIN.C.

tLong idata dos[3]

INSTRUMENT CONTROL Dosimeter Telemetry Application (Main module) (Version 2.0: Flight Software)

This code implements the full INSTRUMENT CONTROL dosimeter sensor, including all message formatting, data processing, and utility functions (ADC, CRC, ...)

Version Rev. 1.0 Created from the code written by Brian Zufelt for the development board and by code written by me for the EU model.

Chip type : Aeroflex UTMC UT69RH051 Program type : Firmware Core Clock frequency : $16.129000 \mathrm{MHz}$

Approved for Public Release; Distribution is unlimited 
Definition at line 33 of file MAIN.C. bit Dos1TurnOn

Definition at line 85 of file MAIN.C. bit Dos2TurnOn

Definition at line 86 of file MAIN.C. bit Dos3TurnOn

Definition at line 87 of file MAIN.C. tWord idata dos_livetime[3]

Definition at line 37 of file MAIN.C. tLong idata dos_zero[3]

Definition at line 34 of file MAIN.C. bit GSE

Definition at line 82 of file MAIN.C. tByte idata HourCount MCF_type idata MCF

Definition at line 80 of file MAIN.C. bit ReadAlllsRunning

Definition at line 81 of file MAIN.C. bit INSTRUMENT CONTROL_Enable

Definition at line 50 of file MAIN.C. bit RunOnce

Definition at line 84 of file MAIN.C.

Approved for Public Release; Distribution is unlimited 
tLong idata RxTimer

Definition at line 60 of file MAIN.C.

Status1_type idata Status1

Definition at line 78 of file MAIN.C.

Status2_type idata Status2

Definition at line 79 of file MAIN.C.

bit TelemetryTick

Definition at line 46 of file MAIN.C.

tLong idata TickCount

Definition at line 57 of file MAIN.C.

tLong idata TotalTickCount

Definition at line 58 of file MAIN.C.

Approved for Public Release; Distribution is unlimited 
// Dosimeter.c : Dosimeter-related function implementations

\#include "main.h"

\#define CAL_PULSE_WIDTH 17

extern Status1_type idata Status1;

extern Status2_type idata Status2;

extern MCF_type idata MCF;

extern tLong idata dos[3];

extern tLong idata dos_zero[3];

extern tLong idata cal_pre[3];

extern tLong idata cal_post[3];

extern tWord idata dos_livetime[3];

extern thong idata TickCount;

extern tLong idata TotalTickCount;

extern tByte idata HourCount;

extern bit TelemetryTick;

extern bit ADC_Start;

extern bit CalRunning;

extern bit ReadAlllsRunning;

extern bit CalMode;

extern bit RunOnce;

extern bit GSE;

extern bit INSTRUMENT CONTROL_Enable;

extern bit Dos1TurnOn;

extern bit Dos2TurnOn;

extern bit Dos3TurnOn;

extern tLong idata RxTimer;

void Init_INSTRUMENT CONTROL( void )

\{

$$
\begin{aligned}
& \text { // } 76543210 \\
& \mathrm{P} 1=0 \times 7 \mathrm{~F} ; \quad \text { // } 01111111 \\
& \text { // +-+-+-- Dosimeters powered off } \\
& \text { // +---+------ ADCs 1,2 disabled } \\
& \text { // +------ SCLK high } \\
& \text { // +------------- MISO set for input (high) } \\
& \text { /l +-------- MOSI set low }
\end{aligned}
$$

// 76543210

// ---- ----

$\mathrm{P} 3=\mathrm{P} 3 \mid 0 \times 2 \mathrm{C} ; \quad / / \mathrm{XX1} \times 11 \times \mathrm{X}$

// +-- RxD controlled by 8051

Approved for Public Release; Distribution is unlimited 


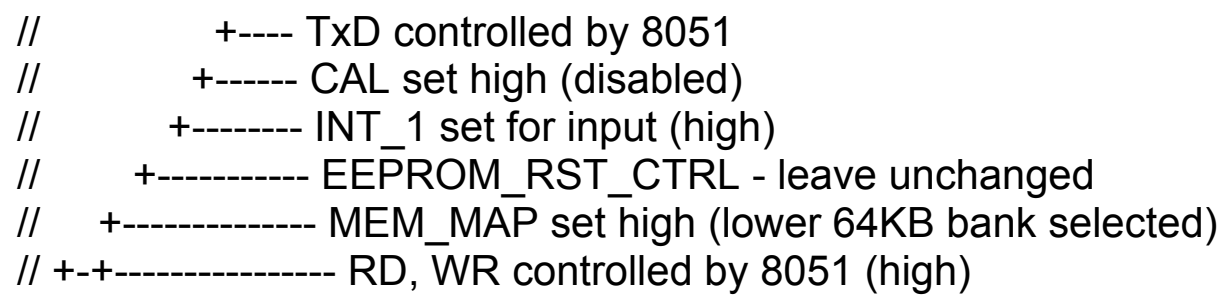

// We leave the "Final" bit set for every packet

Final $=1$;

\}

void SysTimerSetupFast( void )

\{

// Timer 2 is to be used as a system tick timer (in the final system)

// If we use this timer to generate a tick every $2 \mathrm{msec}$, that will

// require a reload value that is 65536 - $(0.002$ seconds * $\mathrm{Tclk})$.

// For a $16.129 \mathrm{MHz}$ oscillator, Finst $=16129000 / 12=1.344083 \mathrm{MHz}$

// This results in Tinst $=744 \mathrm{~ns}$. Therefore $1 \mathrm{~ms}=1,344$ instructions.

// For a system timer interval of $M$ msecs, we need $M * 1344$.

// For $4 \mathrm{~ms}$, the reload timer needs to be $65536-5376=60160$.

// In hexadecimal, this is: 0xEB00

ET2 = 0;

$\mathrm{TR} 2=0$;

$\mathrm{T} 2 \mathrm{CON}=0 \times 04 ; \quad / /$ Load the $\mathrm{T} 2$ timer control register:

// T2 Run on, no mode bits to sweat

T2MOD $=0 \times 00 ; / /$ Timer mode control

$\mathrm{TH} 2=0 \times \mathrm{xB} ; \quad / /$ Load the upper byte

RCAP2H = 0xEB; // Upper byte of the reload value

TL2 = 0x00; // Load the lower byte

RCAP2L $=0 \times 00 ; \quad / /$ Load the lower byte

// Enable the T2 interrupt

$\mathrm{ET} 2 \quad=1$;

// Start the timer running

TR2 = 1;

\}

void SysTimerSetupSlow( void )

\{

// Timer 2 is to be used as a system tick timer (in the final system)

// If we use this timer to generate a tick every $20 \mathrm{msec}$, that will

// require a reload value that is $65536-(0.02$ seconds * $\mathrm{Tclk})$.

// For a $16.129 \mathrm{MHz}$ oscillator, Finst $=16129000 / 12=1.344083 \mathrm{MHz}$

Approved for Public Release; Distribution is unlimited 
// This results in Tinst $=744 \mathrm{~ns}$. Therefore $1 \mathrm{~ms}=1,344$ instructions.

// For a system timer interval of $M$ msecs, we need $M * 1344$.

// For $32 \mathrm{~ms}$, the reload timer needs to be $65536-43,008=22,528$.

// In hexadecimal, this is: $0 \times 5800$

$\mathrm{ET} 2=0$;

TR2 = 0

$\mathrm{T} 2 \mathrm{CON}=0 \times 04 ; \quad / /$ Load the T2 timer control register:

// T2 Run on, no mode bits to sweat

T2MOD = 0x00; // Timer mode control

$\mathrm{TH} 2=0 \times 58$; // Load the upper byte

RCAP2H $=0 \times 58$; // Upper byte of the reload value

$\mathrm{TL2}=0 \times 00$; / Load the lower byte

RCAP2L $=0 \times 00 ; \quad / /$ Load the lower byte

// Enable the T2 interrupt

$\mathrm{ET} 2$ = 1;

// Start the timer running

TR2 = 1;

\}

void SystemTick(void) interrupt INTERRUPT_T2_Overflow

\{

// Clear the T2 overflow flag

$\mathrm{TF} 2=0$;

// Increment the tick count

if (!INSTRUMENT CONTROL_Enable) return;

if $($ AnalysisMode $==0)\{$

TickCount $+=2$;

TotalTickCount $+=2$;

\} else \{

TickCount $+=16$;

TotalTickCount $+=16$; \}

// Increment each livetime counter once every 8.7381333333 minutes.

if (dos1_pwr) \{

if $(($ TotalTickCount \& 0x0003FFFF $)==0) / / 0000000000000011111111111111$

1111

dos_livetime[0]++;

\} else \{

dos_livetime[0] = 0;

\}

Approved for Public Release; Distribution is unlimited 


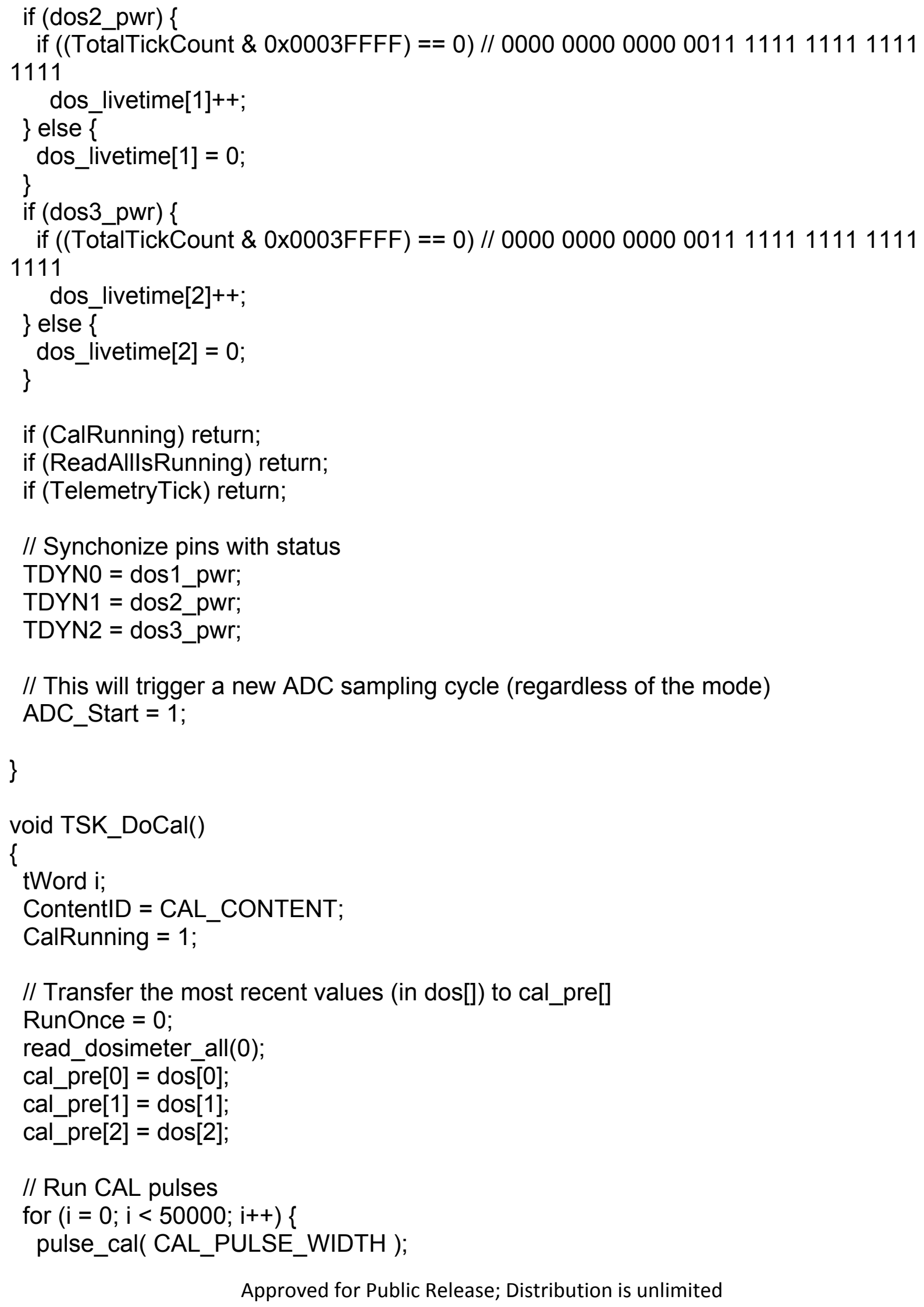


// Set the Cmd history to '0000', VREF Frame to "15V \& 5V"

// Clear CmdRcvd and CmdValid flags.

Status2. value $=0 \times 00$;

// Read the data from the three dosimeters

RunOnce $=0$; // Want to start at the beginning of the aux ADC channels

read_dosimeter_all(0);

cal_post $[0]=$ dos[0];

cal_post[1] = dos[1];

cal_post[2] = dos[2];

// End of Calibration process

CalRunning = 0;

\}

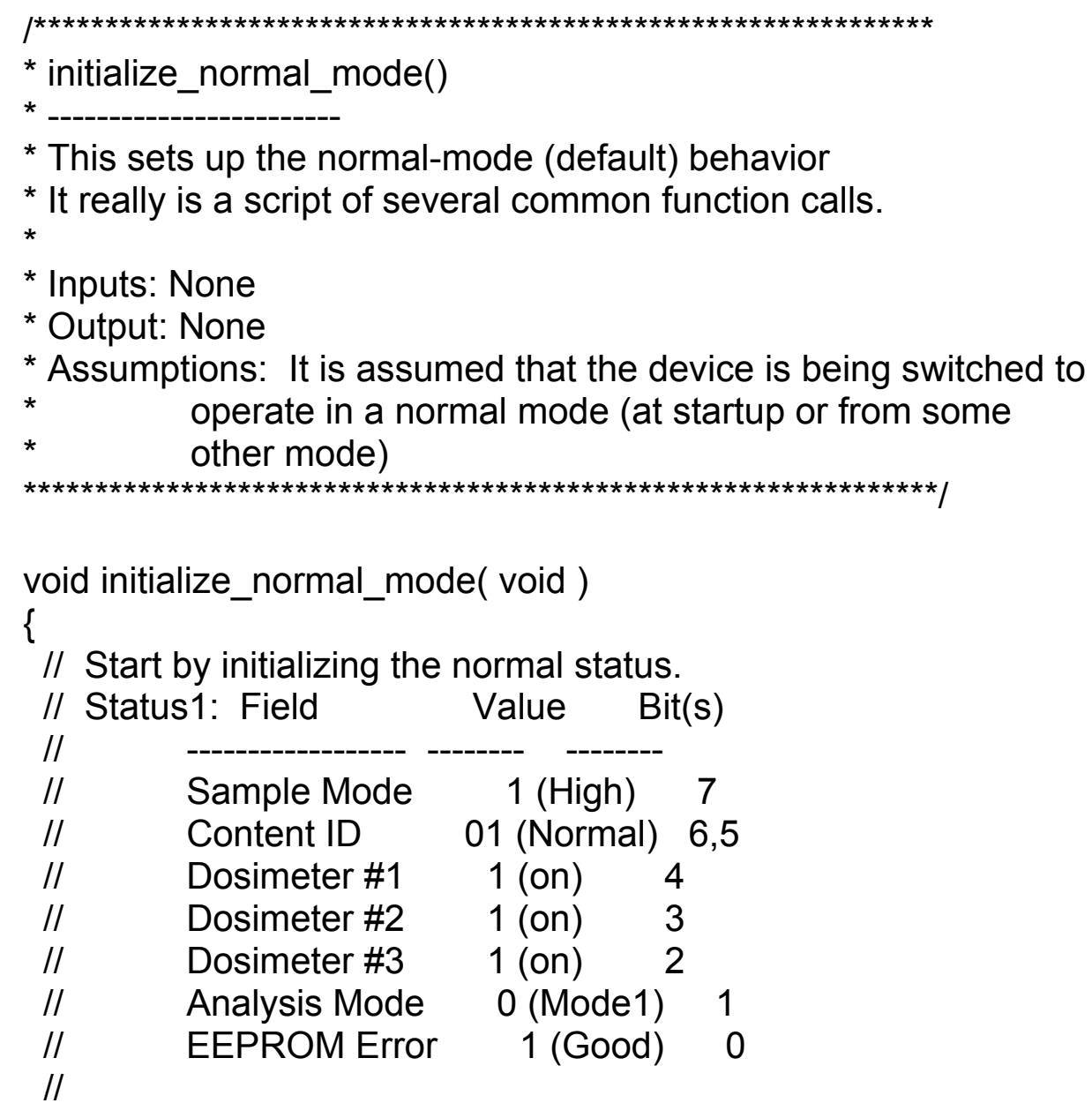




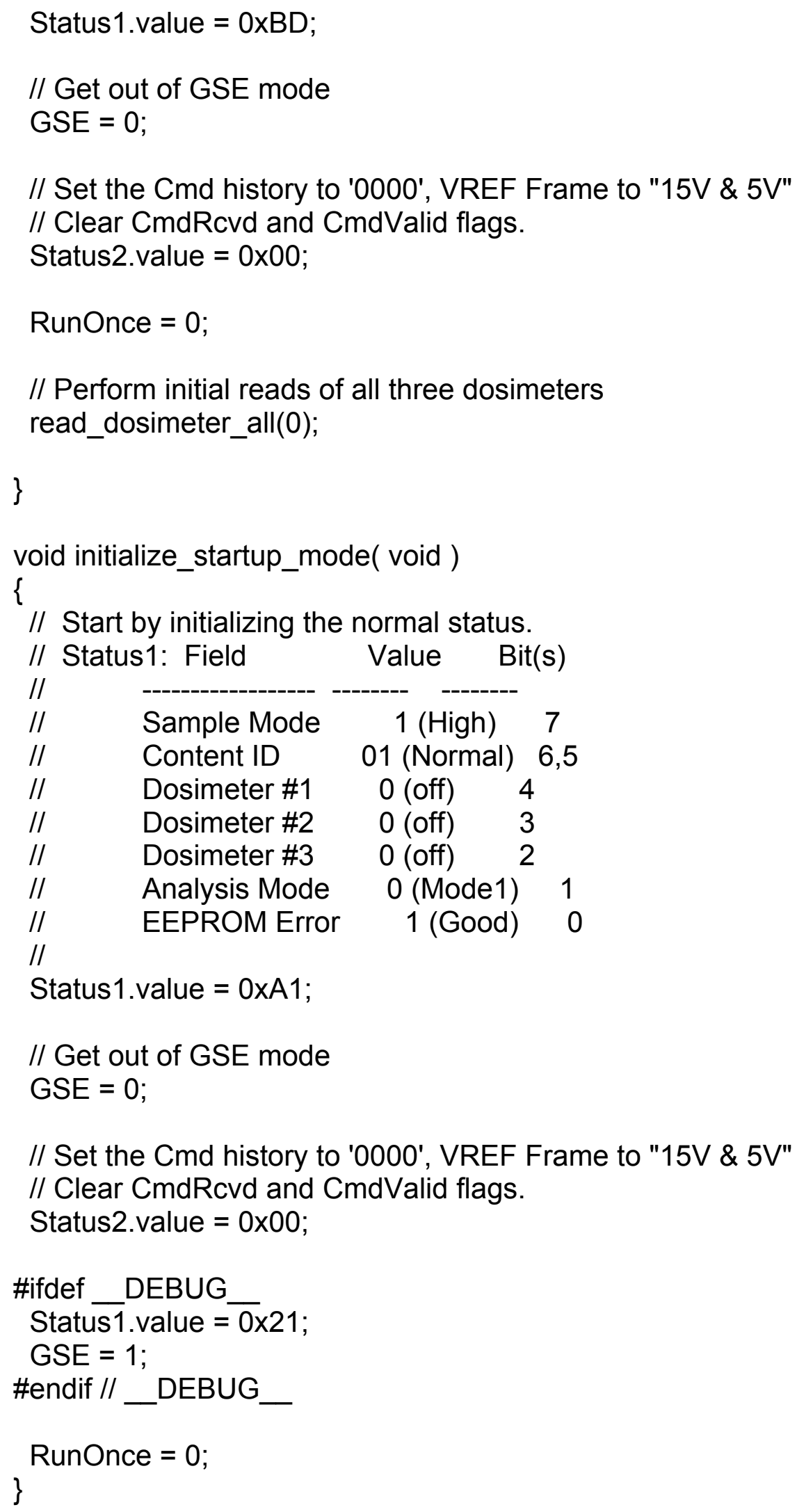




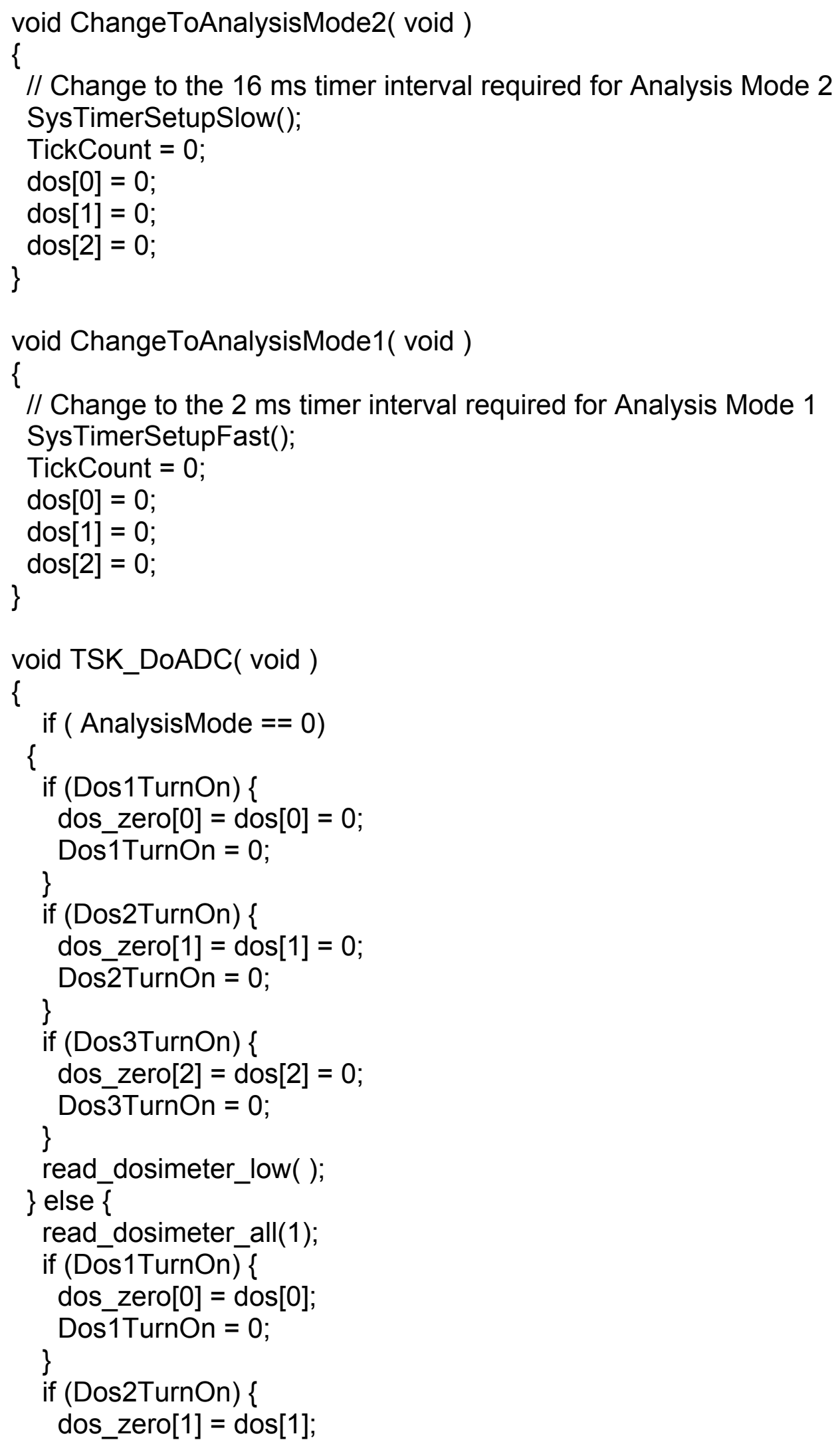

Approved for Public Release; Distribution is unlimited 


\section{Dos2TurnOn = 0; \\ \} \\ if (Dos3TurnOn) \{ \\ dos_zero[2] = dos[2]; \\ Dos3TurnOn = 0;}

Approved for Public Release; Distribution is unlimited 
Interface.c File Reference

\#include "main.h"

Functions

- tWord crc16 (unsigned char *pData, unsigned char len)

- unsigned int SLIP (unsigned char *packet, unsigned int Packet_len)

- unsigned int UNSLIP (unsigned char *packet, unsigned int Packet_len)

\section{Function Documentation}

tWord crc16 (unsigned char * pData, unsigned char len)

Definition at line 25 of file Interface.c.

unsigned int SLIP (unsigned char * packet, unsigned int Packet_len)

Definition at line 84 of file Interface.c.

unsigned int UNSLIP (unsigned char * packet, unsigned int Packet_len)

Definition at line 122 of file Interface.c.

Approved for Public Release; Distribution is unlimited 
Project : INSTRUMENT CONTROL Dosimeter (Interface.c)

Version : $0.1 \mathrm{EU}$ (Engineering Unit)

Date : 2/20/2013

Author : Brian Zufelt

Company : COSMIAC/UNM

Comments:

This source provides all the functions to format the output messages through the serial interface.

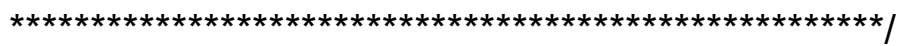

\#include "main.h"

Send a pointer to an array and returns a 16-bit CRC Check.

Return: 16-bit CRC value

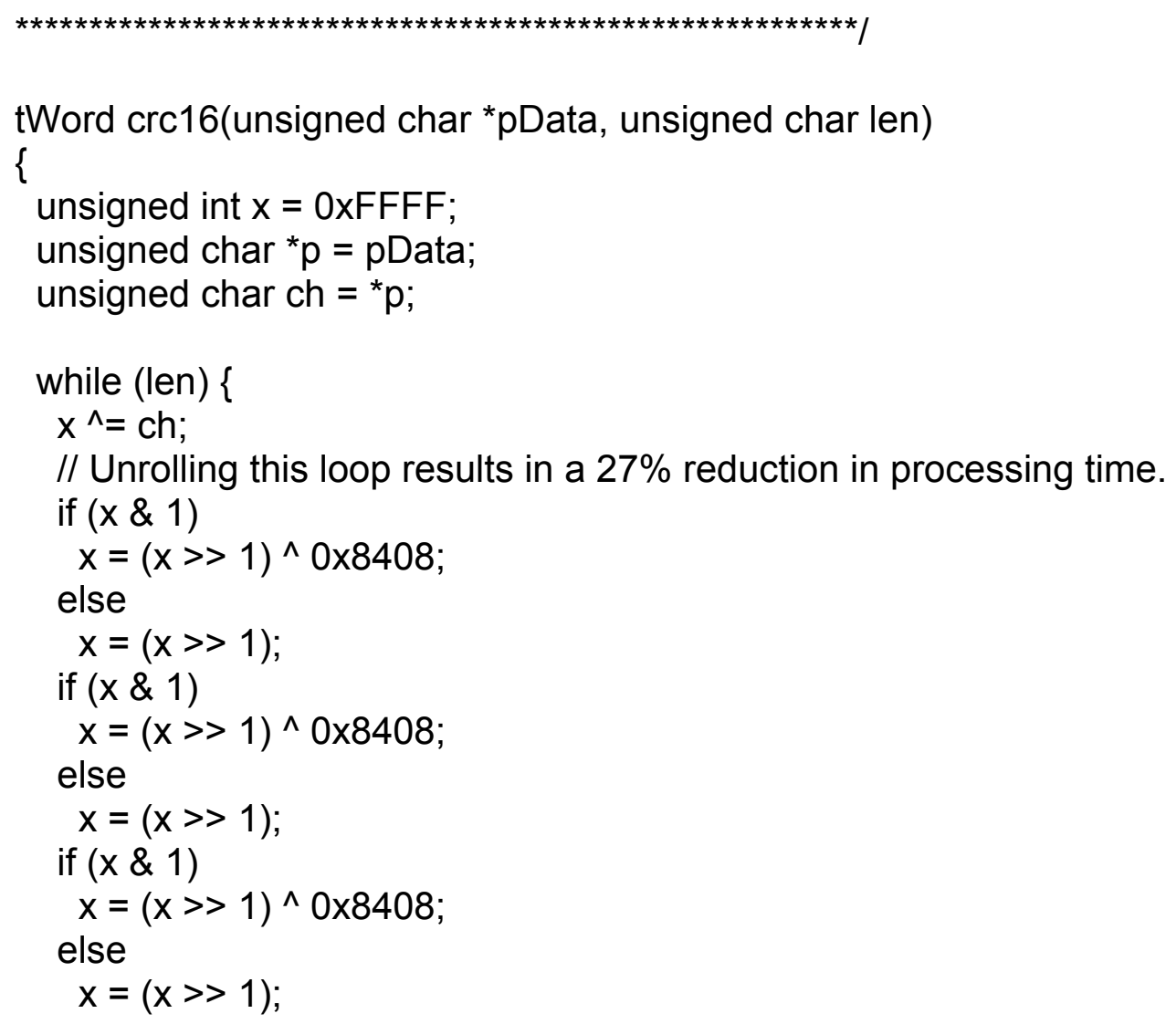

Approved for Public Release; Distribution is unlimited 


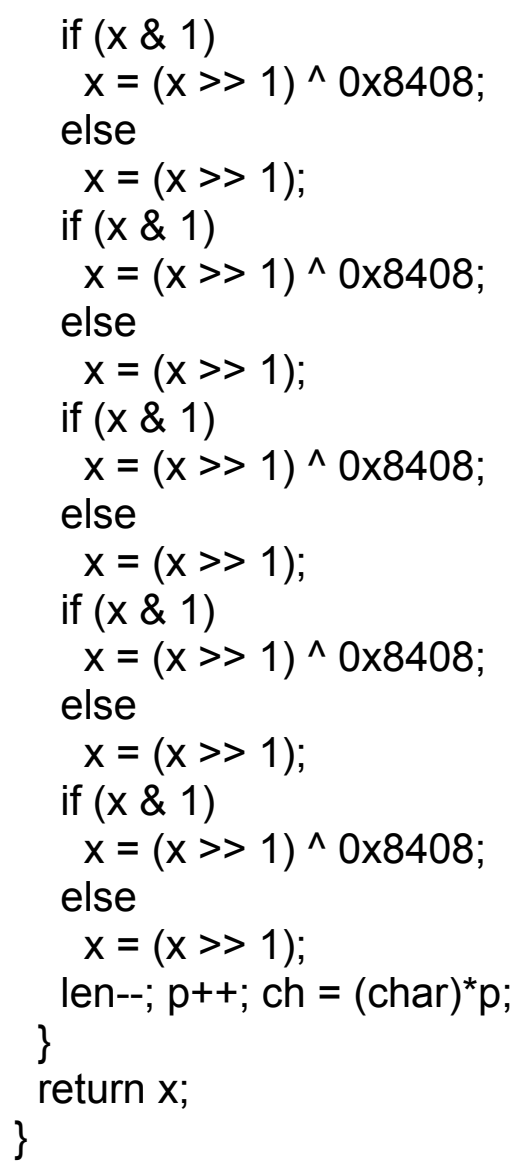

The SLIP Function is Encapsulates the SLIP framing to a given packet.

Returns: New packet length

NOTE: At least packet length +2 bytes are required for start/stop char. Additional size requirements of the buffer depend on the occurance of FEND or FESC within the data packet.

unsigned int SLIP(unsigned char * packet, unsigned int Packet_len)\{

counter

$$
\text { int } \mathrm{i}=0, \mathrm{j}=0 \text {; }
$$

//general

$$
\text { for(i=Packet_len;i>0;i--) \{ }
$$

right

Approved for Public Release; Distribution is unlimited 
\}

$$
\operatorname{packet}[i]=\operatorname{packet}[i-1]
$$

Packet_len $+=2$;

//increase packet size by 2 to add new characters packet[0] = FEND;

/ladd start char

$$
\text { packet[Packet_len-1] = FEND; } \quad \text { //add end char }
$$

for(i=(Packet_len-2);i>1;i--) $\quad / / \mathrm{i}<1$ because packet[0] has to == FEND and packet[Packet_len] $==$ FEND

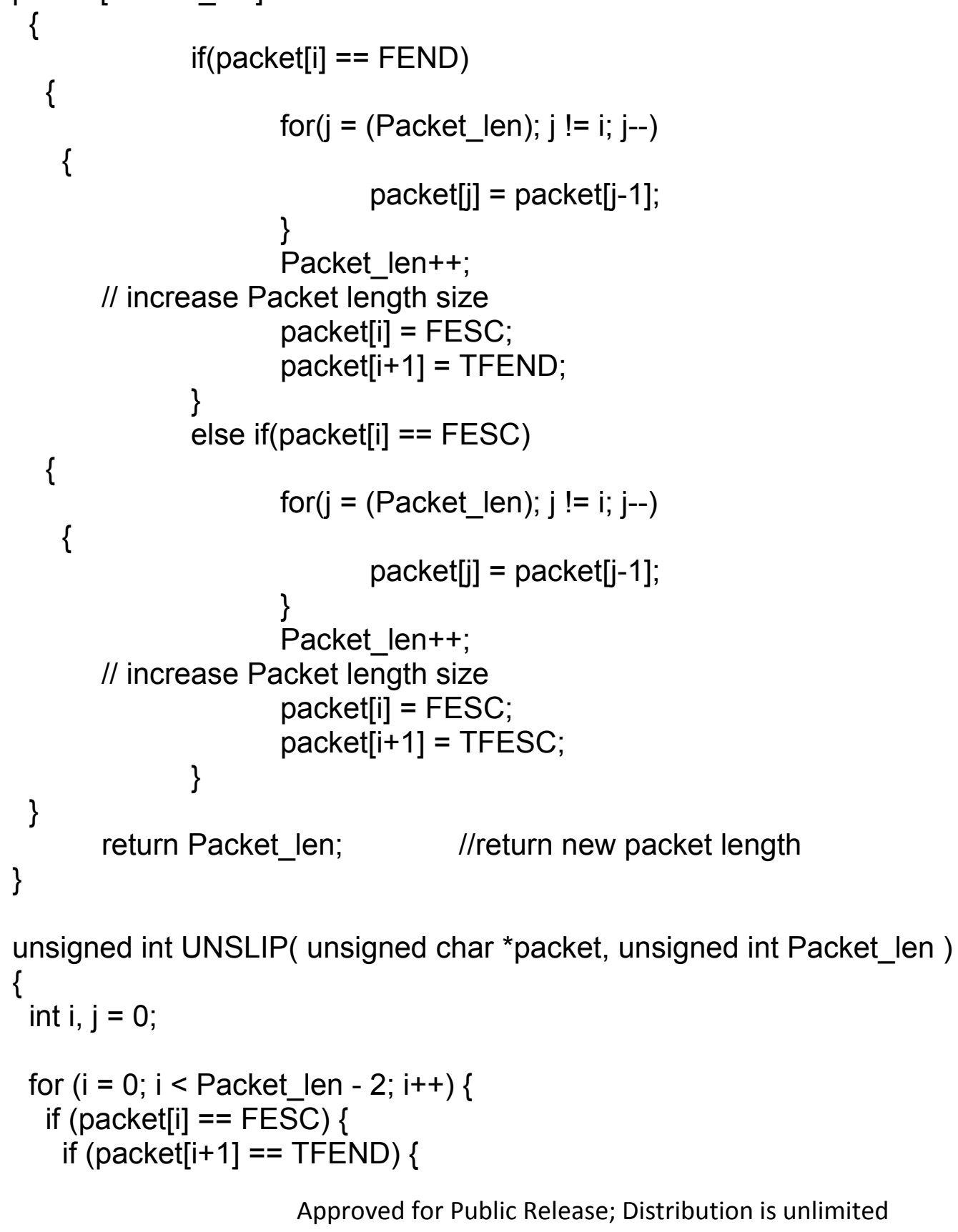

Approved for Public Release; Distribution is unlimited 


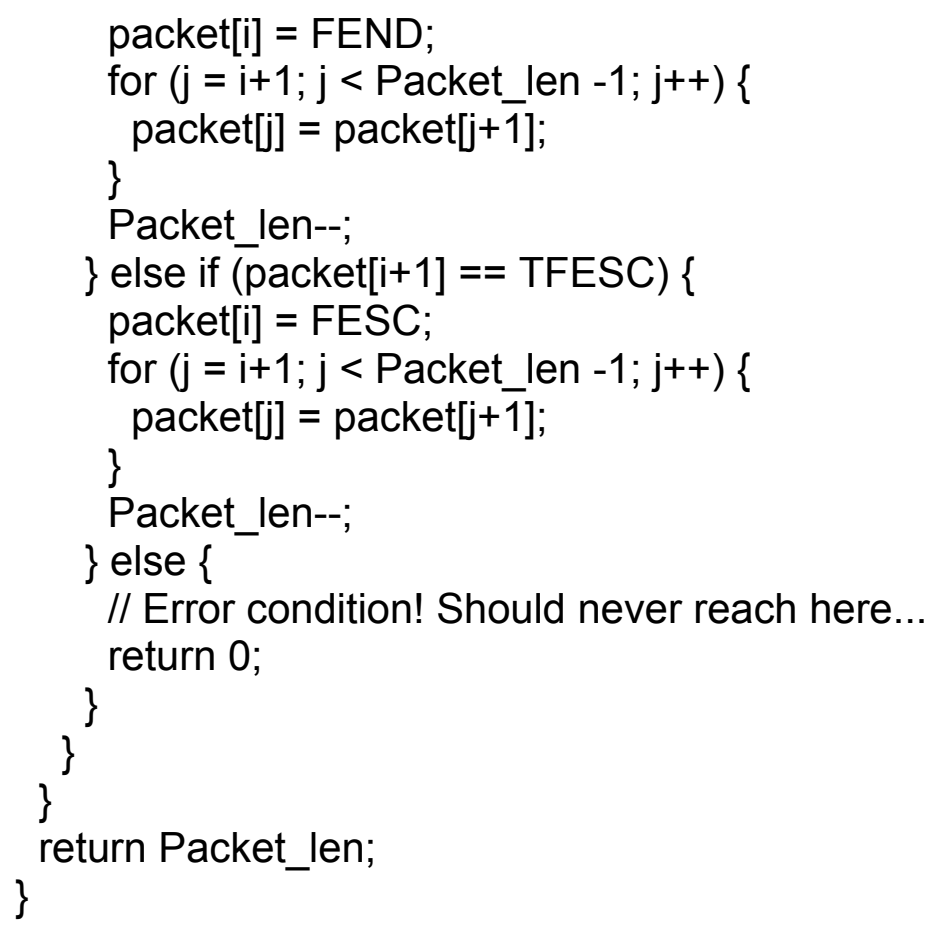

Approved for Public Release; Distribution is unlimited 
MAIN.C File Reference

\#include "main.h"

\section{Functions}

- void main (void)

\section{Variables}

- $\quad{ }^{*}$ tLong idata dos [3] = 0

INSTRUMENT CONTROL Dosimeter Telemetry Application (Main module) (Version

2.0: Flight Software)

- tLong idata dos_zero [3] = 0

- tLong idata cal_pre [3] = 0

- tLong idata cal_post [3] = 0

- tWord idata dos_livetime [3] = 0

- tWord idata ps_15V

- tWord idata ps_5V

- tWord idata temps [3]

- tWord idata FrameCounter $=0$

- bit TelemetryTick = 0

- bit ADC_Start =0

- bit ResetImminent $=0$

- $\quad$ bit TxEnable = 0

- $\quad$ bit INSTRUMENT CONTROL_Enable = 0

- bit CalRunning = 0

- $\quad$ bit CalMode = 1

- $\quad$ tLong idata TickCount $=0$

- $\quad$ tLong idata TotalTickCount $=0$

- tWord idata PktCnt_Cal =0

- $\quad$ tLong idata RxTimer = 0xFFFFFFFF

- $\quad$ Byte idata XmitLength $=0$

- tByte idata DataBuff [48]

- tByte idata AckBuff [14]

- tByte idata * pXmitPtr

- tByte idata RecvLength $=6$

- MessageQueue msgQueue

- tByte idata RecvBuff [10]

- tByte idata * pRecvPtr

- Status1_type idata Status1

- Status2_type idata Status2

- MCF_type idata MCF

- bit ReadAllIsRunning = 0

- bit GSE = 0

- bit Poll = 0 
- bit RunOnce $=0$

- bit Dos1TurnOn =0

- bit Dos2TurnOn =0

- bit Dos3TurnOn = 0

- tByte code adc_ch_mode2 [3][5]

- tByte code adc_ch []

\section{Function Documentation}

void main (void)

Definition at line 108 of file MAIN.C.

\section{Variable Documentation}

tByte idata AckBuff[14]

Definition at line 65 of file MAIN.C.

tByte code adc_ch $]$

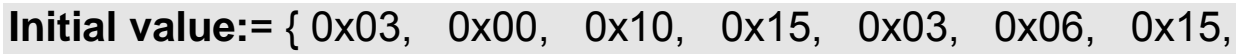
$0 \times 00, \quad 0 \times 03, \quad 0 \times 00, \quad 0 \times 11,0 \times 15,0 \times 03,0 \times 00$, $0 \times 12, \quad 0 \times 15, \quad 0 \times 03, \quad 0 \times 00,0 \times 14,0 \times 15\}$

Definition at line 104 of file MAIN.C.

tByte code adc_ch_mode2[3][5]

Initial value:=

\{

$\{0 \times 00,0 \times 06,0 \times 00,0 \times 00,0 \times 00\}$,

$\{0 \times 10,0 \times 15,0 \times 11,0 \times 12,0 \times 14\}$,

\}

$\{0 \times 15,0 \times 00,0 \times 15,0 \times 15,0 \times 15\}$

Definition at line 91 of file MAIN.C.

bit $A D C$ Start $=0$

Definition at line 47 of file MAIN.C.

tLong idata cal_post[3] = 0

Definition at line 36 of file MAIN.C.

Approved for Public Release; Distribution is unlimited 
tLong idata cal_pre[3] $=0$

Definition at line 35 of file MAIN.C.

bit CalMode $=1$

Definition at line 54 of file MAIN.C.

bit CalRunning $=0$

Definition at line 53 of file MAIN.C.

tByte idata DataBuff[48]

Definition at line 64 of file MAIN.C.

* tLong idata dos[3] = 0

INSTRUMENT CONTROL Dosimeter Telemetry Application (Main module) (Version 2.0: Flight Software)

This code implements the full INSTRUMENT CONTROL dosimeter sensor, including all message formatting, data processing, and utility functions (ADC, CRC, ...)

Version Rev. 1.0 Created from the code written by Brian Zufelt for the development board and by code written by me for the EU model.

Chip type : Aeroflex UTMC UT69RH051 Program type : Firmware Core Clock frequency : $16.129000 \mathrm{MHz}$

Definition at line 33 of file MAIN.C.

bit Dos1TurnOn $=0$

Definition at line 85 of file MAIN.C.

bit Dos2TurnOn = 0

Definition at line 86 of file MAIN.C.

bit Dos3TurnOn $=0$

Definition at line 87 of file MAIN.C.

Approved for Public Release; Distribution is unlimited 
tWord idata dos_livetime[3] $=0$

Definition at line 37 of file MAIN.C.

tLong idata dos_zero[3] $=0$

Definition at line 34 of file MAIN.C.

tWord idata FrameCounter $=0$

Definition at line 43 of file MAIN.C.

bit GSE = 0

Definition at line 82 of file MAIN.C.

MCF_type idata MCF

Definition at line 80 of file MAIN.C.

MessageQueue msgQueue

Definition at line 68 of file MAIN.C.

tWord idata PktCnt_Cal $=0$

Definition at line 59 of file MAIN.C.

bit Poll = 0

Definition at line 83 of file MAIN.C.

tByte idata* pRecvPtr

Definition at line 75 of file MAIN.C.

tWord idata ps_15V

Definition at line 38 of file MAIN.C.

tWord idata ps_5V

Definition at line 39 of file MAIN.C.

Approved for Public Release; Distribution is unlimited 
tByte idata* XXmitPtr

Definition at line 66 of file MAIN.C.

bit ReadAlllsRunning $=0$

Definition at line 81 of file MAIN.C.

tByte idata RecvBuff[10]

Definition at line 73 of file MAIN.C.

tByte idata RecvLength $=6$

Definition at line 67 of file MAIN.C.

bit ResetImminent $=0$

Definition at line 48 of file MAIN.C.

bit INSTRUMENT CONTROL_Enable $=0$

Definition at line 50 of file MAIN.C.

bit RunOnce $=0$

Definition at line 84 of file MAIN.C.

tLong idata RxTimer $=0 x F F F F F F F F$

Definition at line 60 of file MAIN.C.

Status1_type idata Status1

Definition at line 78 of file MAIN.C.

Status2_type idata Status2

Definition at line 79 of file MAIN.C.

bit TelemetryTick $=0$

Definition at line 46 of file MAIN.C.

Approved for Public Release; Distribution is unlimited 
tWord idata temps[3]

Definition at line 40 of file MAIN.C.

tLong idata TickCount $=0$

Definition at line 57 of file MAIN.C.

tLong idata TotalTickCount $=0$

Definition at line 58 of file MAIN.C.

bit TXEnable $=0$

Definition at line 49 of file MAIN.C.

tByte idata XmitLength $=0$

Definition at line 63 of file MAIN.C.

$/ / !$

//! Ibrief INSTRUMENT CONTROL Dosimeter Telemetry Application (Main module)

I/! (Version 2.0: Flight Software)

$/ / !$

I/! This code implements the full INSTRUMENT CONTROL dosimeter sensor,

//! including all message formatting, data processing, and

//! utility functions (ADC, CRC, ...)

//!

//! Version Rev.

//! 1.0 Created from the code written by Brian Zufelt for

I/! the development board and by code written by me

//! for the EU model.

I/!

//! Chip type $\quad$ : Aeroflex UTMC UT69RH051

//! Program type : Firmware

//! Core Clock frequency $\quad: 16.129000 \mathrm{MHz}$

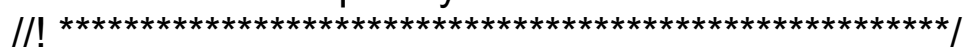

Approved for Public Release; Distribution is unlimited 


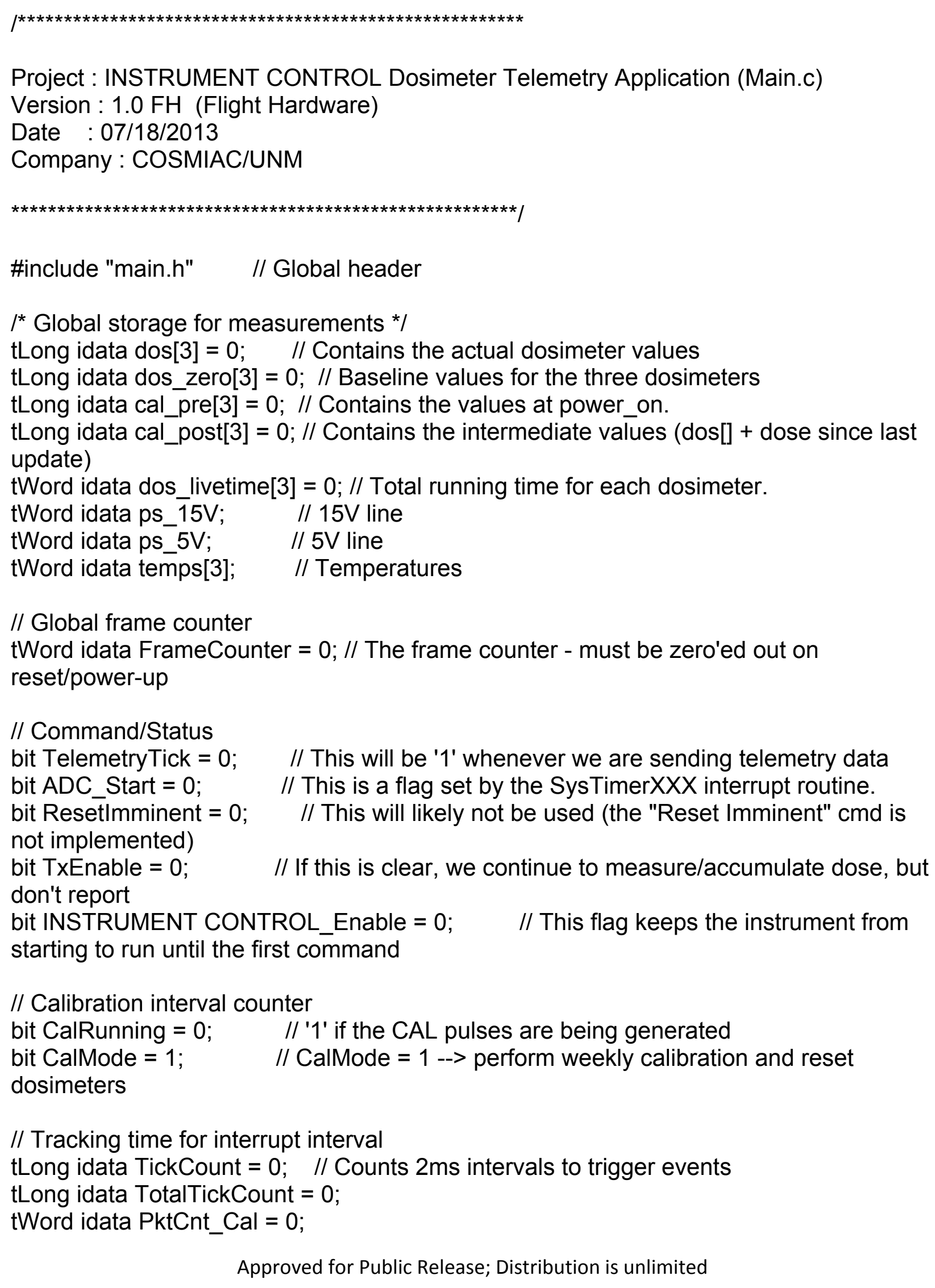


tLong idata RxTimer = 0xFFFFFFFF; // Initialize to high end of range

// Serial comm buffers

tByte idata XmitLength =0; // If this value is non-zero, there is data to transmit

tByte idata DataBuff[48]; // Data block containing chars to be xmit'd

tByte idata AckBuff[14]; // Data block for Ack packet chars

tByte idata * $\mathrm{pXmitPtr}$ / // Pointer for iterating through the xmit buffer

tByte idata RecvLength $=6$; // Command receive length

MessageQueue msgQueue; // Contains the pointers and lengths for data / ack

packets

\#ifdef _DEBUG

tByte idata RecvBuff[10] $=\{0 \times 56,0 \times 11,0 \times A 2,0 \times 7 E, 0 \times 44,0 \times 52,0 \times 00,0 \times 00,0 \times 00$, $0 \times 00\}$;

\#else

tByte idata RecvBuff[10];

\#endif // DEBUG

tByte idata *pRecvPtr;

// Status bytes and Command/Status flags

Status1_type idata Status1;

Status2_type idata Status2;

MCF_type idata MCF;

bit ReadAlllsRunning $=0$;

bit GSE = 0;

bit Poll = 0;

bit RunOnce = 0;

bit Dos1TurnOn =0;

bit Dos2TurnOn =0;

bit Dos3TurnOn = 0;

// This 2D array contains the ADC dev/ch sequence for Analysis Mode 2

// (The first index is the "stage", the second is where we are in <count>)

tByte code adc_ch_mode2[3][5] =

$$
\begin{aligned}
& \{/(1)(2)(3)(4)(5) \\
& \quad\{0 \times 00,0 \times 06,0 \times 00,0 \times 00,0 \times 00\}, \quad / / \text { "Stage 0" } \\
& \quad\{0 \times 10,0 \times 15,0 \times 11,0 \times 12,0 \times 14\}, \quad / / \text { "Stage 1" } \\
& \{0 \times 15,0 \times 00,0 \times 15,0 \times 15,0 \times 15\} \quad / / \text { "Stage 2" } \\
& \} ;
\end{aligned}
$$

// This 1D array contains the ADC dev/ch sequence for Analysis Mode 1

// (There are four measurements per timer tick:
/I
//
//
Dos1Low,
Dos2Low,
Dos3Low,

Approved for Public Release; Distribution is unlimited 
tByte code adc_ch[] $=\{0 \times 03, \quad 0 \times 00,0 \times 10,0 \times 15,0 \times 03,0 \times 06,0 \times 15$, $0 \times 00, \quad 0 \times 03,0 \times 00,0 \times 11,0 \times 15,0 \times 03,0 \times 00$,

0x12, $\quad 0 \times 15,0 \times 03,0 \times 00,0 \times 14,0 \times 15\}$;

void main(void)\{

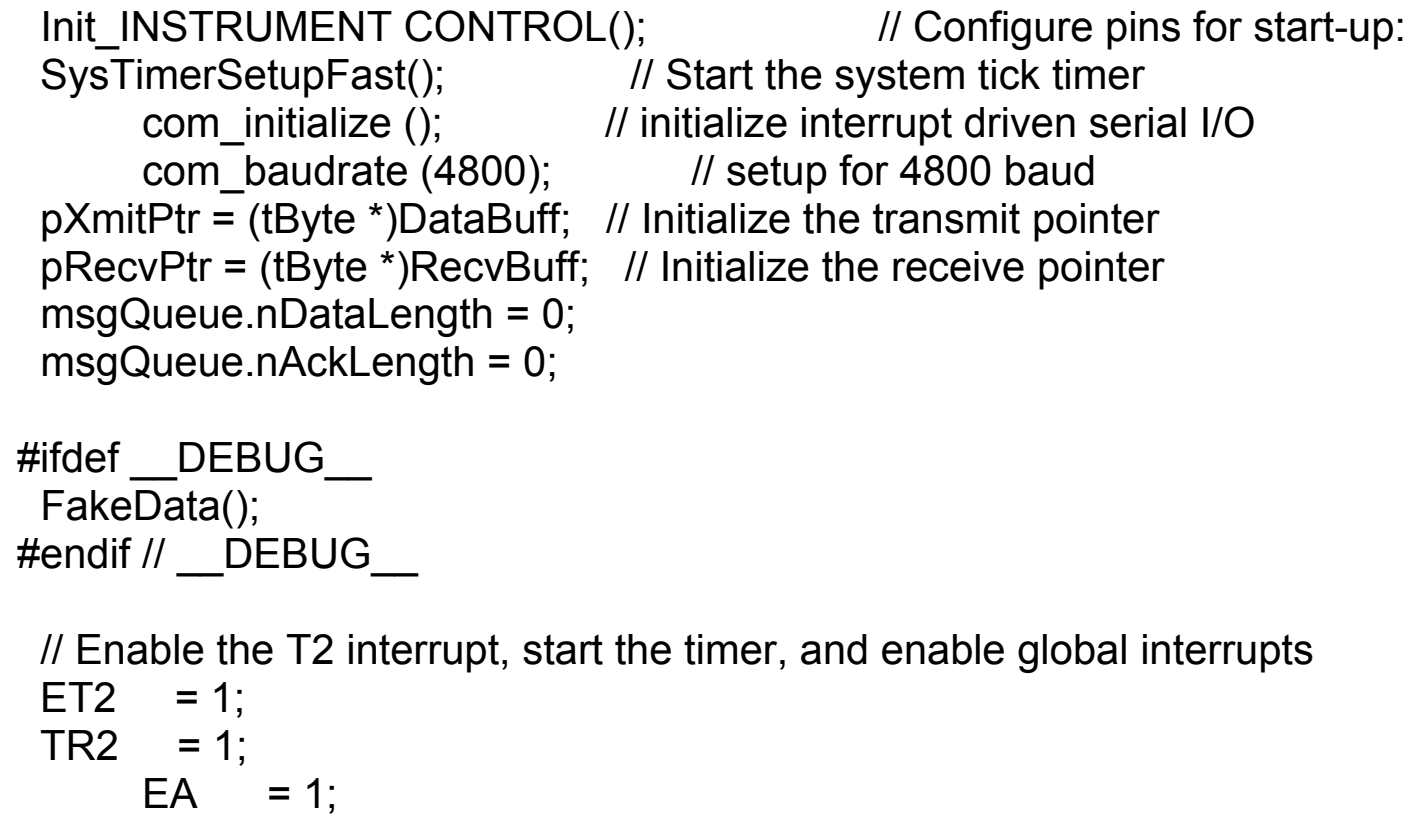

// Make sure the instrument comes up in an inactive, controlled state initialize_startup_mode();

TickCount = 0;

RunOnce = 0;

// Debug only \#ifdef _DEBUG //printf("Write this to the serial port...Irln"); \#endif // _ DEBUG

\#ifdef __DEBUG

RecvLength $=6$; // Length of received message...

TSK_DoCmdParse(); \#endif $/ /$ DEBUG

while (1) \{

// This is the main execution loop that cycles forever.

// 1. Check if it's time to create a new telemetry packet.

TelemetryTick = TimeToXmitTelemetry(); 


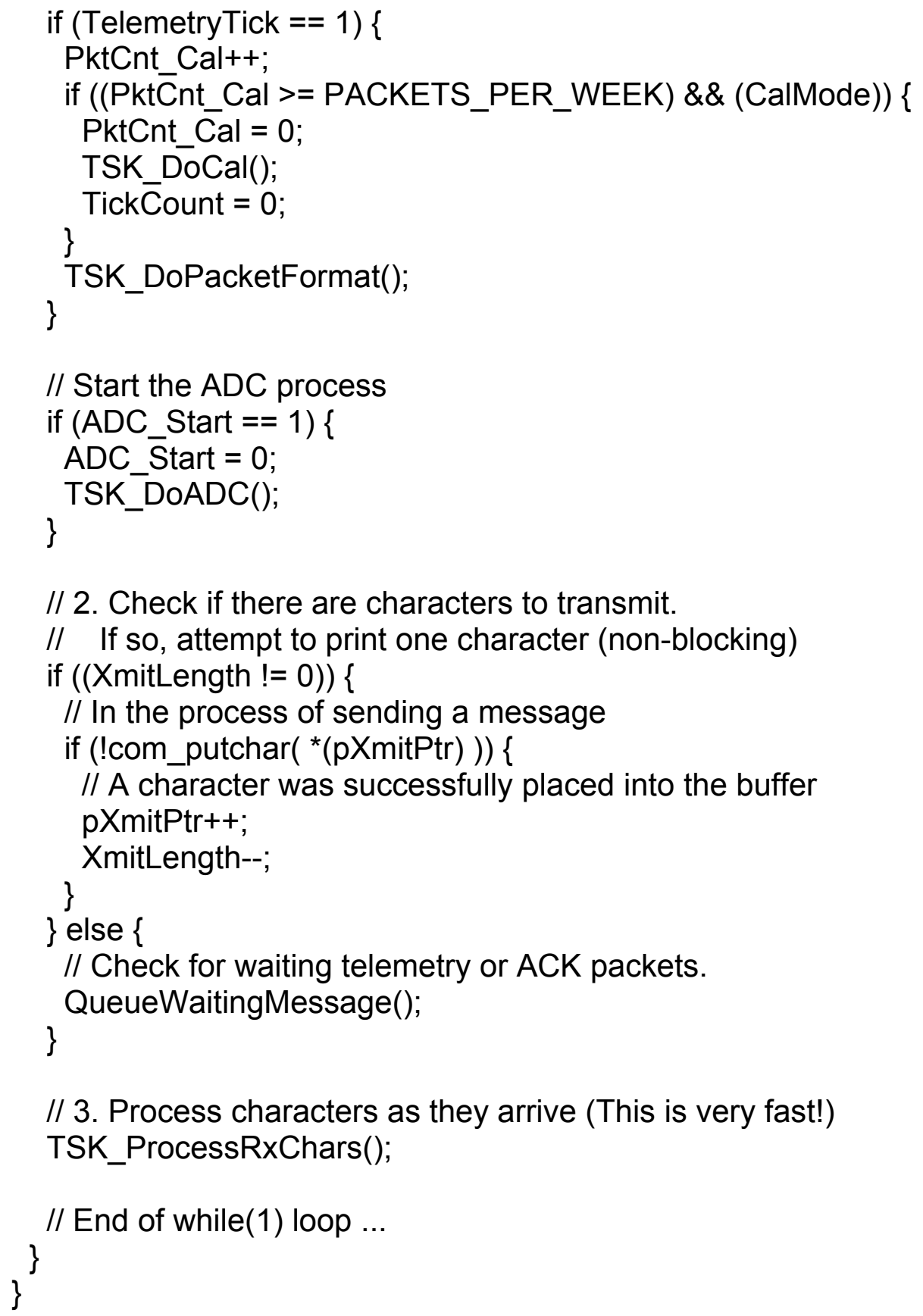

// 2. Check if there are characters to transmit.

// If so, attempt to print one character (non-blocking)

if $(($ XmitLength ! $=0))\{$

// In the process of sending a message

if (!com_putchar( *(pXmitPtr) )) \{

// A character was successfully placed into the buffer pXmitPtr++;

// 3. Process characters as they arrive (This is very fast!) TSK_ProcessRxChars();

// End of while(1) loop ... \} 


\section{Packet.c File Reference}

\#include "main.h"

\section{Functions}

- void TSK_ProcessRxChars (void)

- void TSK_DoCmdParse (void)

- void TSK_DoAckPacket (void)

- void TSK_DoNakPacket (tByte nak_code)

- void TSK_DoPacketFormat (void)

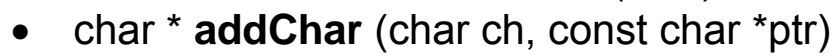

- char * addWord (tWord val, const char *ptr)

- char $^{*}$ addLong (tLong val, const char *ptr)

- tByte TimeToXmitTelemetry (void)

- void QueueWaitingMessage (void)

\section{Variables}

- MCF_type idata MCF

- tLong idata dos [3] INSTRUMENT CONTROL Dosimeter Telemetry Application (Main module) (Version 2.0: Flight Software)

- tLong idata dos_zero[3]

- tLong idata cal_pre[3]

- tLong idata cal_post [3]

- tWord idata dos_livetime [3]

- tWord idata ps_15V

- tWord idata ps_5V

- tWord idata temps [3]

- tWord idata FrameCounter

- bit CalMode

- tLong idata TickCount

- tLong idata TotalTickCount

- bit TelemetryTick

- tByte idata XmitLength

- tByte idata DataBuff [48]

- tByte idata AckBuff [14]

- Status1_type idata Status1

- Status2_type idata Status2

- tByte idata * pXmitPtr

- tByte idata * pRecvPtr

- tByte idata RecvLength

- tByte idata RecvBuff [10]

- bit GSE

- bit Poll

- bit TxEnable 
- bit INSTRUMENT CONTROL_Enable

- bit ResetImminent

- bit Dos1TurnOn

- bit Dos2TurnOn

- bit Dos3TurnOn

- tLong idata RxTimer

- tWord idata PktCnt_Cal

- MessageQueue msgQueue

\section{Function Documentation}

char* addChar (char ch, const char * ptr)

Definition at line 455 of file Packet.c.

char* addLong (tLong val, const char * ptr)

Definition at line 468 of file Packet.c.

char* addWord (tWord val, const char * ptr)

Definition at line 462 of file Packet.c.

void QueueWaitingMessage (void )

Definition at line 516 of file Packet.c.

tByte TimeToXmitTelemetry (void)

Definition at line 474 of file Packet.c.

void TSK_DoAckPacket (void)

Definition at line 265 of file Packet.c.

void TSK_DoCmdParse (void )

Definition at line 108 of file Packet.c.

void TSK_DoNakPacket (tByte nak_code)

Definition at line 283 of file Packet.c.

Approved for Public Release; Distribution is unlimited 
void TSK_DoPacketFormat (void)

Definition at line 303 of file Packet.c.

void TSK_ProcessRxChars (void)

Definition at line 42 of file Packet.c.

\section{Variable Documentation}

tByte idata AckBuff[14]

Definition at line 65 of file MAIN.C.

tLong idata cal_post[3]

Definition at line 36 of file MAIN.C.

tLong idata cal_pre[3]

Definition at line 35 of file MAIN.C.

bit CalMode

Definition at line 54 of file MAIN.C.

tByte idata DataBuff[48]

Definition at line 64 of file MAIN.C.

tLong idata dos[3]

INSTRUMENT CONTROL Dosimeter Telemetry Application (Main module) (Version 2.0: Flight Software)

This code implements the full INSTRUMENT CONTROL dosimeter sensor, including all message formatting, data processing, and utility functions (ADC, CRC, ...)

Version Rev. 1.0 Created from the code written by Brian Zufelt for the development board and by code written by me for the EU model.

Chip type : Aeroflex UTMC UT69RH051 Program type : Firmware Core Clock frequency : $16.129000 \mathrm{MHz}$

Approved for Public Release; Distribution is unlimited 
Definition at line 33 of file MAIN.C. bit Dos1TurnOn

Definition at line 85 of file MAIN.C. bit Dos2TurnOn

Definition at line 86 of file MAIN.C. bit Dos3TurnOn

Definition at line 87 of file MAIN.C. tWord idata dos_livetime[3]

Definition at line 37 of file MAIN.C. tLong idata dos_zero[3]

Definition at line 34 of file MAIN.C. tWord idata FrameCounter

Definition at line 43 of file MAIN.C. bit GSE

Definition at line 82 of file MAIN.C. MCF_type idata MCF

Definition at line 80 of file MAIN.C. MessageQueue msgQueue

Definition at line 68 of file MAIN.C. tWord idata PktCnt_Cal

Definition at line 59 of file MAIN.C. bit Poll 
Definition at line 83 of file MAIN.C. tByte idata* $p$ RecvPtr

Definition at line 75 of file MAIN.C. tWord idata ps_15V

Definition at line 38 of file MAIN.C. tWord idata ps_5V

Definition at line 39 of file MAIN.C. tByte idata* $p$ XmitPtr

Definition at line 66 of file MAIN.C. tByte idata RecvBuff[10]

Definition at line 73 of file MAIN.C. tByte idata RecvLength

Definition at line 67 of file MAIN.C. bit Resetlmminent

Definition at line 48 of file MAIN.C. bit INSTRUMENT CONTROL_Enable

Definition at line 50 of file MAIN.C. tLong idata RxTimer

Definition at line 60 of file MAIN.C.

Status1_type idata Status1

Definition at line 78 of file MAIN.C. Status2_type idata Status2

Approved for Public Release; Distribution is unlimited 
Definition at line 79 of file MAIN.C.

bit TelemetryTick

Definition at line 46 of file MAIN.C.

tWord idata temps[3]

Definition at line 40 of file MAIN.C.

tLong idata TickCount

Definition at line 57 of file MAIN.C.

tLong idata TotalTickCount

Definition at line 58 of file MAIN.C.

bit TXEnable

Definition at line 49 of file MAIN.C.

tByte idata XmitLength

Definition at line 63 of file MAIN.C.

Approved for Public Release; Distribution is unlimited 
// Packet.c

// This file contains the code for assembling packets for transmittal to ARCS

\#include "main.h"

extern MCF_type idata MCF;

extern tLong idata dos[3];

extern tLong idata dos_zero[3];

extern tLong idata cal_pre[3];

extern tLong idata cal_post[3];

extern tWord idata dos_livetime[3];

extern tWord idata ps_15V;

extern tWord idata ps_5V;

extern tWord idata temps[3];

extern tWord idata FrameCounter;

extern bit CalMode;

extern tLong idata TickCount;

extern tLong idata TotalTickCount;

extern bit TelemetryTick;

extern tByte idata XmitLength;

extern tByte idata DataBuff[48];

extern tByte idata AckBuff[14];

extern Status1_type idata Status1;

extern Status2_type idata Status2;

extern tByte idata * $p X m i t P t r$;

extern tByte idata * $p$ RecvPtr;

extern tByte idata RecvLength;

extern tByte idata RecvBuff[10];

extern bit GSE;

extern bit Poll;

extern bit TxEnable;

extern bit INSTRUMENT CONTROL_Enable;

extern bit ResetImminent;

extern bit Dos1TurnOn;

extern bit Dos2TurnOn;

extern bit Dos3TurnOn;

extern tLong idata RxTimer;

extern tWord idata PktCnt_Cal;

extern MessageQueue msgQueue;

// UART task functions

void TSK_ProcessRxChars(void)

\{

// Create the state variable and initialize it to starting state

static RxStateType state = WaitingFirstFEND;

int rx_char;

Approved for Public Release; Distribution is unlimited 


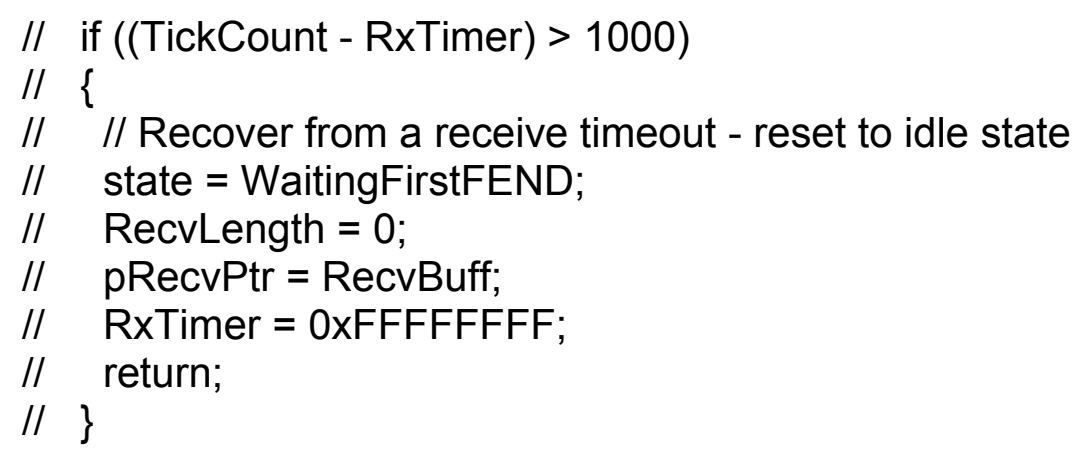

// Check comm port for a character $(-1=$ empty $)$ rx_char = com_getchar(); if $($ rx_char $==-1$ ) return;

//com_putchar(rx_char);

switch(state)

\{ case WaitingFirstFEND:

if $($ ((tByte)rx_char) $==$ FEND)

\{

state $=$ WaitingFirstNonFEND;

RecvLength = 0;

RxTimer = TickCount;

\}

return;

break;

case WaitingFirstNonFEND:

if ( (tByte)rx_char != FEND)

\{

state $=$ ReadingChars;

${ }^{*}($ pRecvPtr $)=($ tByte $)$ rx_char; pRecvPtr++;

RecvLength++;

\}

return;

break;

case ReadingChars:

if $($ (tByte)rx_char $==$ FEND)

\{

state = WaitingFirstFEND;

RxTimer = 0xFFFFFFFF;

// Found the end of the command - now process it

Approved for Public Release; Distribution is unlimited 


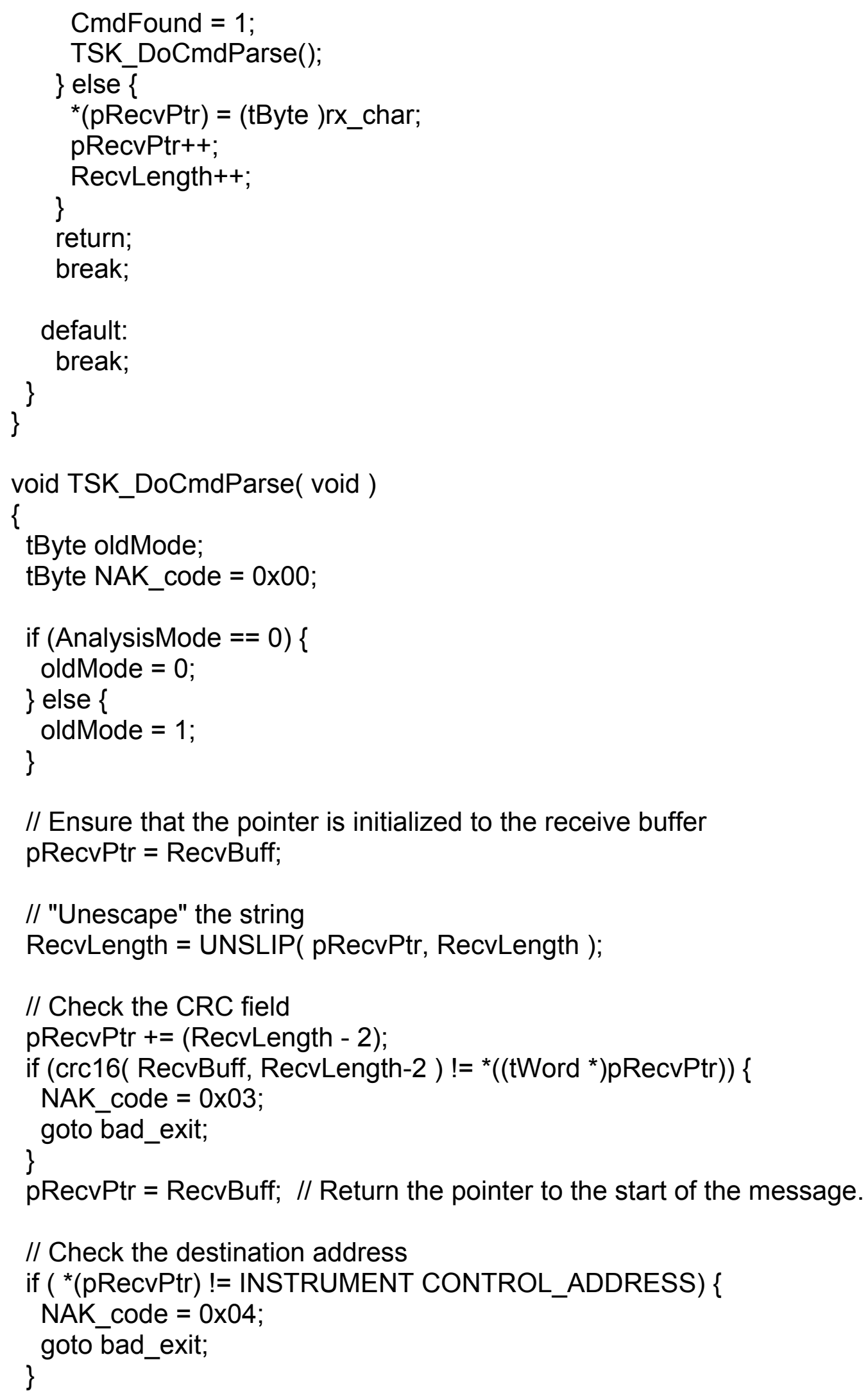

// Ensure that the pointer is initialized to the receive buffer pRecvPtr = RecvBuff;

// "Unescape" the string

Approved for Public Release; Distribution is unlimited 
// Check the source address

pRecvPtr++;

if $\left({ }^{*}(\right.$ pRecvPtr $) !=$ ARCS_ADDRESS $)\{$

NAK_code $=0 \times 05$;

\}

goto bad_exit;

// Assertion: The command is from the avionics

// host and is intended for INSTRUMENT CONTROL

// Parse the message control field

pRecvPtr++;

Poll $=\left({ }^{*}(\right.$ pRecvPtr $\left.) \& 0 \times 80\right)>>7$;

B_bit $=\left({ }^{*}(\right.$ pRecvPtr $\left.) \& 0 \times 40\right)>>6$; // Pass through unaltered - we don't use this bit.

CmdCode $=\left({ }^{*}(\mathrm{pRecvPtr}) \& 0 \times 1 \mathrm{~F}\right)$;

$\mathrm{Cmd}=\left({ }^{*}(\mathrm{pRecvPtr}) \& 0 \mathrm{xOF}\right)$;

if $(\mathrm{Cmd}==0 \times 01)\{/ /$ "Instrument Reset" command (1 payload byte)

if (RecvLength $==6)$ \{

INSTRUMENT CONTROL_Enable $=1$;

pRecvPtr++;

if ( $\left.{ }^{*}(\mathrm{pRecvPtr}) !=0\right)\{/ /$ Reset is imminent - perform an orderly shutdown

ResetImminent $=1$;

\} else \{

ResetImminent $=0$;

\}

goto good_exit;

\} else \{

NAK_code $=0 \times 02$;

goto bad_exit;

\}

\} else if ( $\mathrm{Cmd}==0 \times 02)\{/ /$ "Configure" command ( 1 payload byte)

if (RecvLength $==6)$ \{

INSTRUMENT CONTROL_Enable $=1$;

CmdValid $=1$;

pRecvPtr++;

GSE $=\left({ }^{*}(\mathrm{pRecvPtr}) \& 0 \times 80\right)>>7$;

Status1.fields. SampleMode $=\left({ }^{*}(\right.$ RecvPtr $\left.) \& 0 \times 40\right)>>6$;

if $(($ CalMode $) \& \&(((*$ pRecvPtr $) \& 0 \times 20)>>5)==1))\{$

// If CalMode goes from 0 --> 1 , force a CAL

PktCnt_Cal $=$ PACKETS_PER_WEEK;

TickCount = 0xFFFF0000;

\}

CalMode $=\left({ }^{*}(\right.$ pRecvPtr $\left.) \& 0 \times 20\right)>>5$;

TxEnable $=\left({ }^{*}(\mathrm{pRecvPtr}) \& 0 \times 10\right)>>4$

Approved for Public Release; Distribution is unlimited 
// Test for dos1_pwr switching from off to on

Dos1TurnOn = dos1_pwr;

dos1_pwr = ( *(pRecvPtr) \& 0x08) >> 3;

if ((dos1_pwr) \&\& (!Dos1TurnOn))

Dos1TurnOn = 1;

else

Dos1TurnOn = 0;

// Test for dos2_pwr switching from off to on

Dos2TurnOn = dos2_pwr;

dos2_pwr $=\left({ }^{*}(\right.$ pRecvPtr $\left.) \& 0 \times 04\right)>>2$;

if ((dos2_pwr) \&\& (!Dos2TurnOn))

Dos2TurnOn = 1;

else

Dos2TurnOn = 0;

// Test for dos3_pwr switching from off to on

Dos3TurnOn = dos3_pwr;

dos3_pwr $=\left({ }^{*}(\right.$ pRecvPtr $\left.) \& 0 \times 02\right)>>1$;

if ((dos3_pwr) \&\& (!Dos3TurnOn))

Dos3TurnOn $=1$;

else

Dos3TurnOn = 0;

AnalysisMode $=\left({ }^{*}(\mathrm{pRecvPtr}) \& 0 \times 01\right)$;

// Test to see if the analysis mode has changed ...

// If it has, need to change the system timer tick interval

if $(($ oldMode $==0) \& \&($ AnalysisMode $==1))$

ChangeToAnalysisMode2();

if $(($ oldMode $==1) \& \&($ AnalysisMode $==0)$ )

ChangeToAnalysisMode1();

goto good_exit;

\} else \{

NAK_code $=0 \times 02$;

goto bad_exit;

\}

\} else if $(\mathrm{Cmd}==0 \times 03)\{/ /$ "Normal mode" command ( 0 payload bytes)

if $($ RecvLength $==5)\{$

// Initialize the normal mode of operation

INSTRUMENT CONTROL_Enable $=1$;

// Set the TurnOn flag for each dosimeter that was off

if (!dos1_pwr) Dos1TurnOn = 1;

if (!dos2_pwr) Dos2TurnOn = 1;

if (!dos3_pwr) Dos3TurnOn = 1;

initialize_normal_mode();

// Change to faster timer tick

Approved for Public Release; Distribution is unlimited 


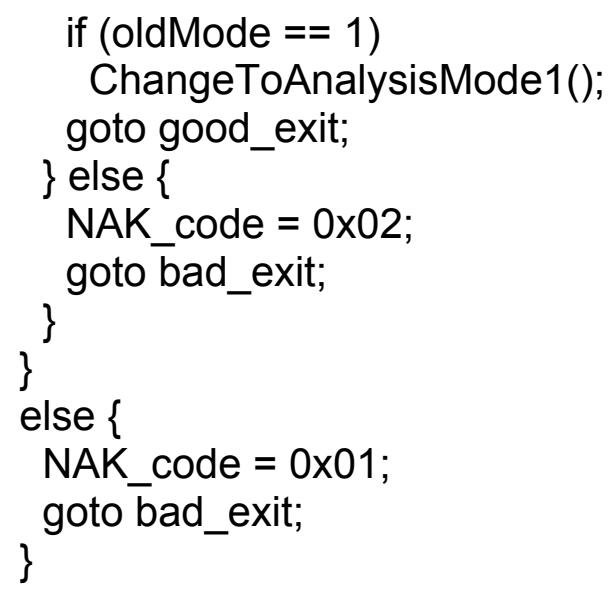

// Don't often use GOTOs, but sometimes they are useful bad_exit:

CmdValid = 0;

Ack = 0;

if (Poll)

TSK_DoNakPacket( NAK_code ); goto exit_func;

good_exit:

CmdValid = 1;

Ack = 1;

if (Poll)

TSK_DoAckPacket();

Ack $=0$;

exit_func:

pRecvPtr $=\left(\right.$ tByte $\left.{ }^{*}\right)$ RecvBuff; return;

\}

// ACK/NAK packet formatting functions void TSK_DoAckPacket( void )

\{

// Start the data insertion point just past the MCF char ${ }^{*}$ ptr = AckBuff +3 ;

tWord crc;

// Pre-populate the values that are always the same AckBuff[0] $=0 \times 11 ; \quad / /$ Destination address $($ host $($ ARCS $)=0 \times 11)$ AckBuff[1] = 0x56; // Source address (INSTRUMENT CONTROL $=0 \times 56$ ) AckBuff[2] = MCF.value; // Message Control Field = // OxAO for most NSP telemetry packets $\operatorname{crc}=\operatorname{crc16}\left(\left(\right.\right.$ char $\left.^{*}\right)($ AckBuff $\left.), 3\right)$;

Approved for Public Release; Distribution is unlimited 


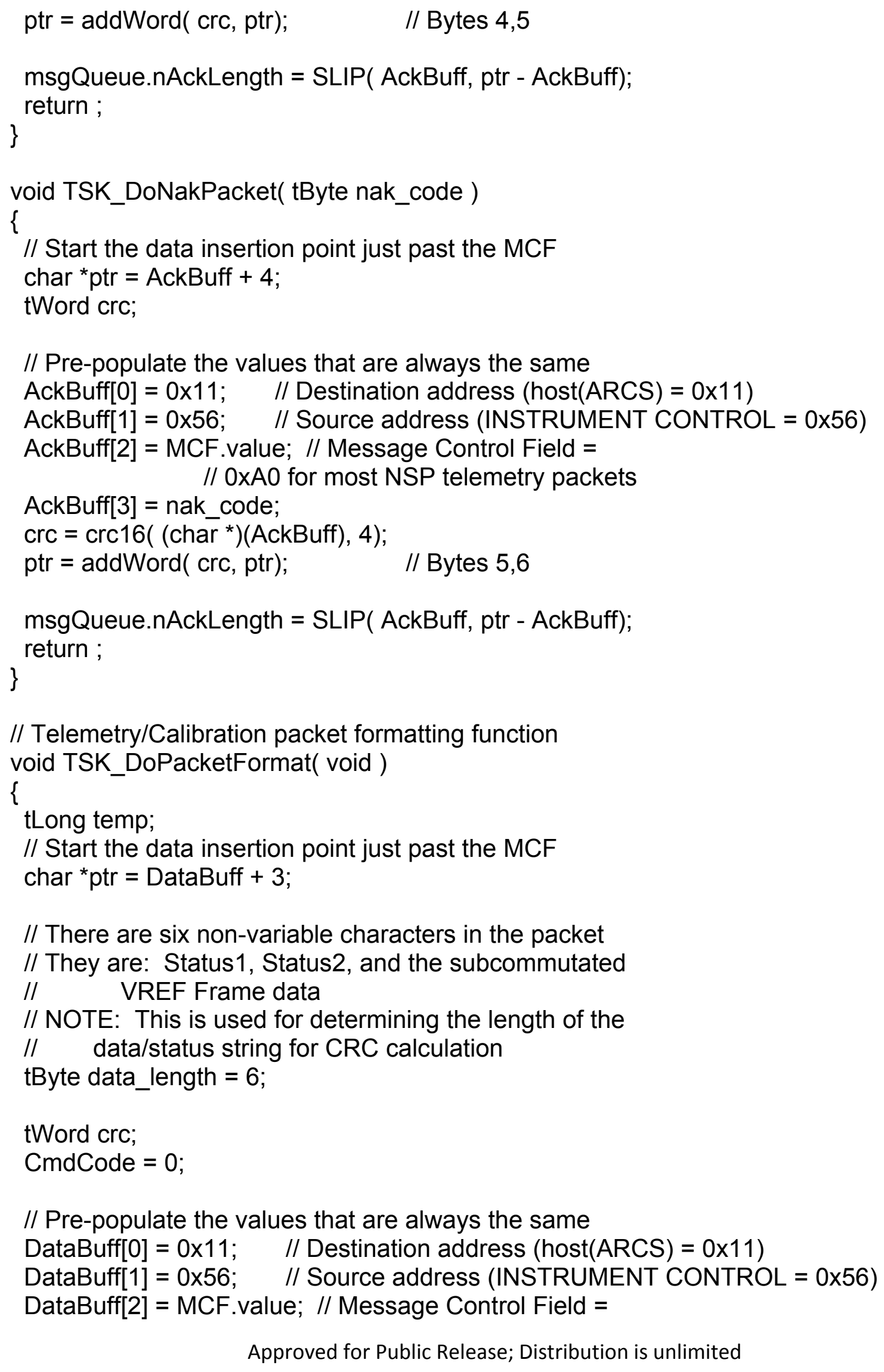

// Telemetry/Calibration packet formatting function void TSK_DoPacketFormat( void )

// Pre-populate the values that are always the same DataBuff $[0]=0 \times 11 ; \quad / /$ Destination address $($ host $($ ARCS $)=0 \times 11)$ DataBuff[1] = 0x56; // Source address (INSTRUMENT CONTROL $=0 \times 56$ ) DataBuff[2] = MCF.value; $/ /$ Message Control Field = 


\section{// 0xA0 for most NSP telemetry packets}

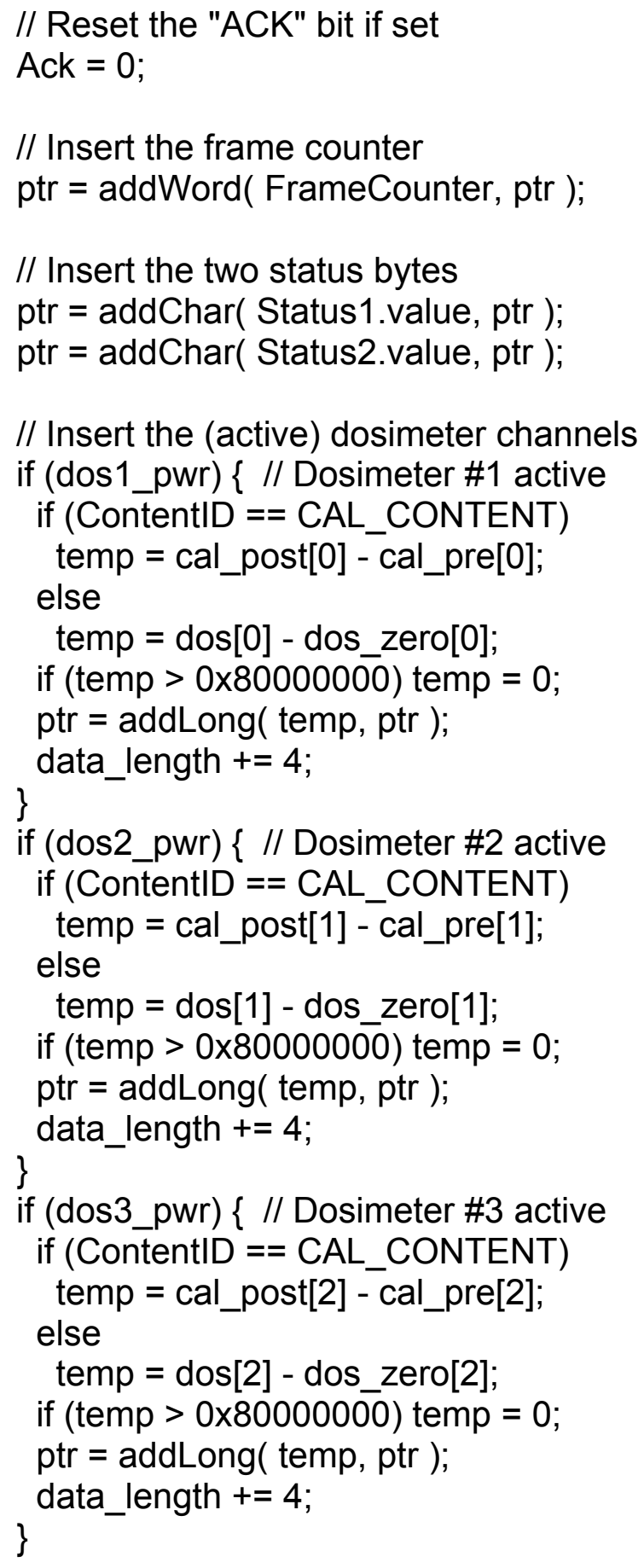

// Reset the flag that indicates that we are in the process of // formatting the buffer string (this prevents ADC sampling during // this period). NOTE: NEED TO CHECK WITH CHAD ON THIS. // Note: This time should be very short, since most of the time // spent in this whole routine is spent later in the CRC calcs. 


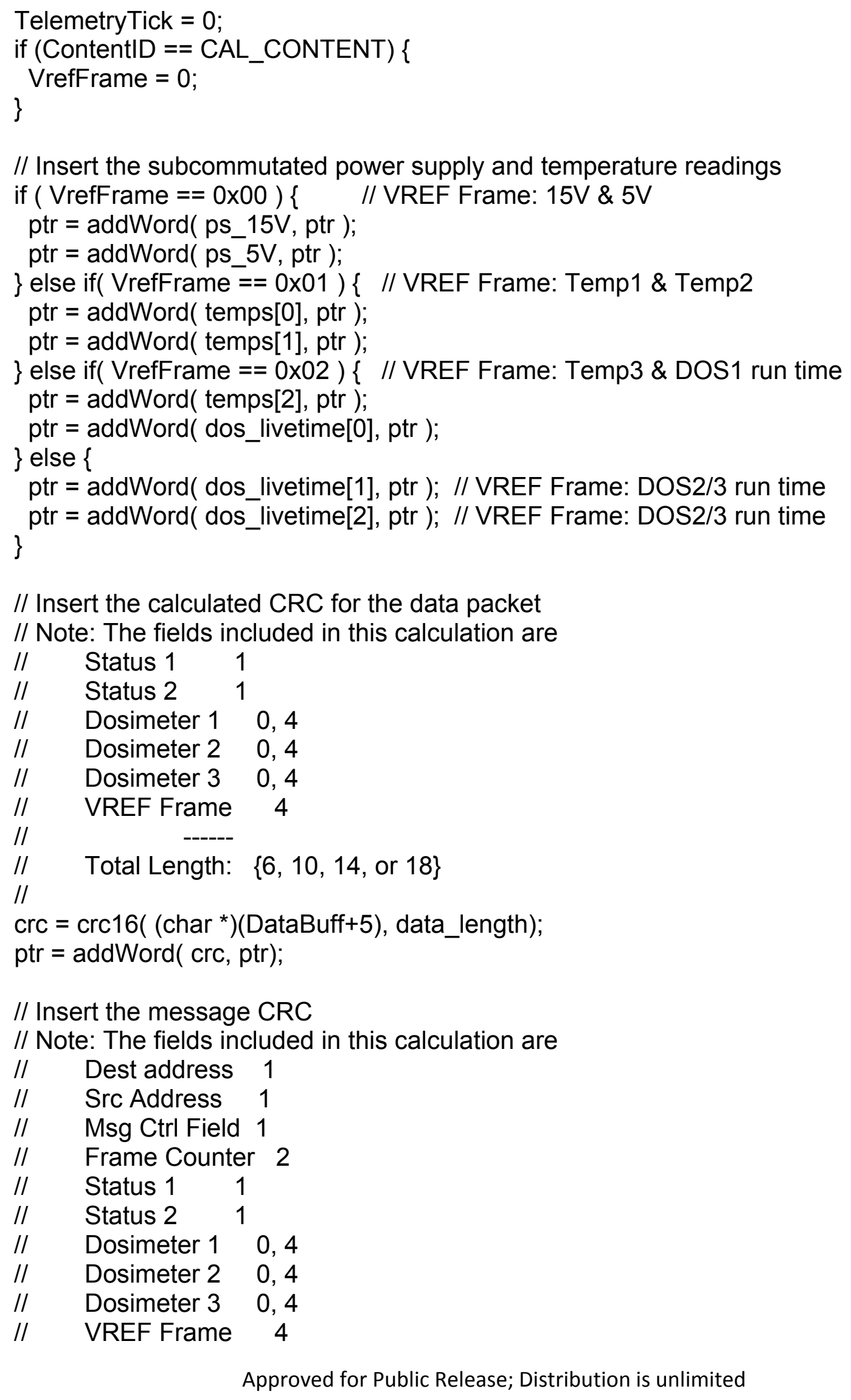

// Insert the calculated CRC for the data packet

// Note: The fields included in this calculation are

// Status $1 \quad 1$

// Status $2 \quad 1$

// Dosimeter $1 \quad 0,4$

// Dosimeter 20,4

// Dosimeter 3 0, 4

/I VREF Frame 4

/I

// Total Length: $\{6,10,14$, or 18$\}$

/I

$\operatorname{crc}=\operatorname{crc16}\left(\left(\right.\right.$ char $\left.^{*}\right)($ DataBuff +5$)$, data_length $) ;$

$\mathrm{ptr}=\operatorname{addWord}(\mathrm{crc}, \mathrm{ptr})$;

// Insert the message CRC

// Note: The fields included in this calculation are

$\begin{array}{lll}\text { /I } & \text { Dest address } & 1 \\ \text { /I } & \text { Src Address } & 1 \\ \text { /I } & \text { Msg Ctrl Field 1 } \\ \text { /I } & \text { Frame Counter } & 2 \\ \text { /I } & \text { Status 1 } 1 \\ \text { /I } & \text { Status 2 } & 1 \\ \text { /I } & \text { Dosimeter 1 } & 0,4 \\ \text { /I } & \text { Dosimeter 2 } & 0,4 \\ \text { /I } & \text { Dosimeter 3 } & 0,4 \\ \text { /I } & \text { VREF Frame } & 4\end{array}$

Approved for Public Release; Distribution is unlimited 


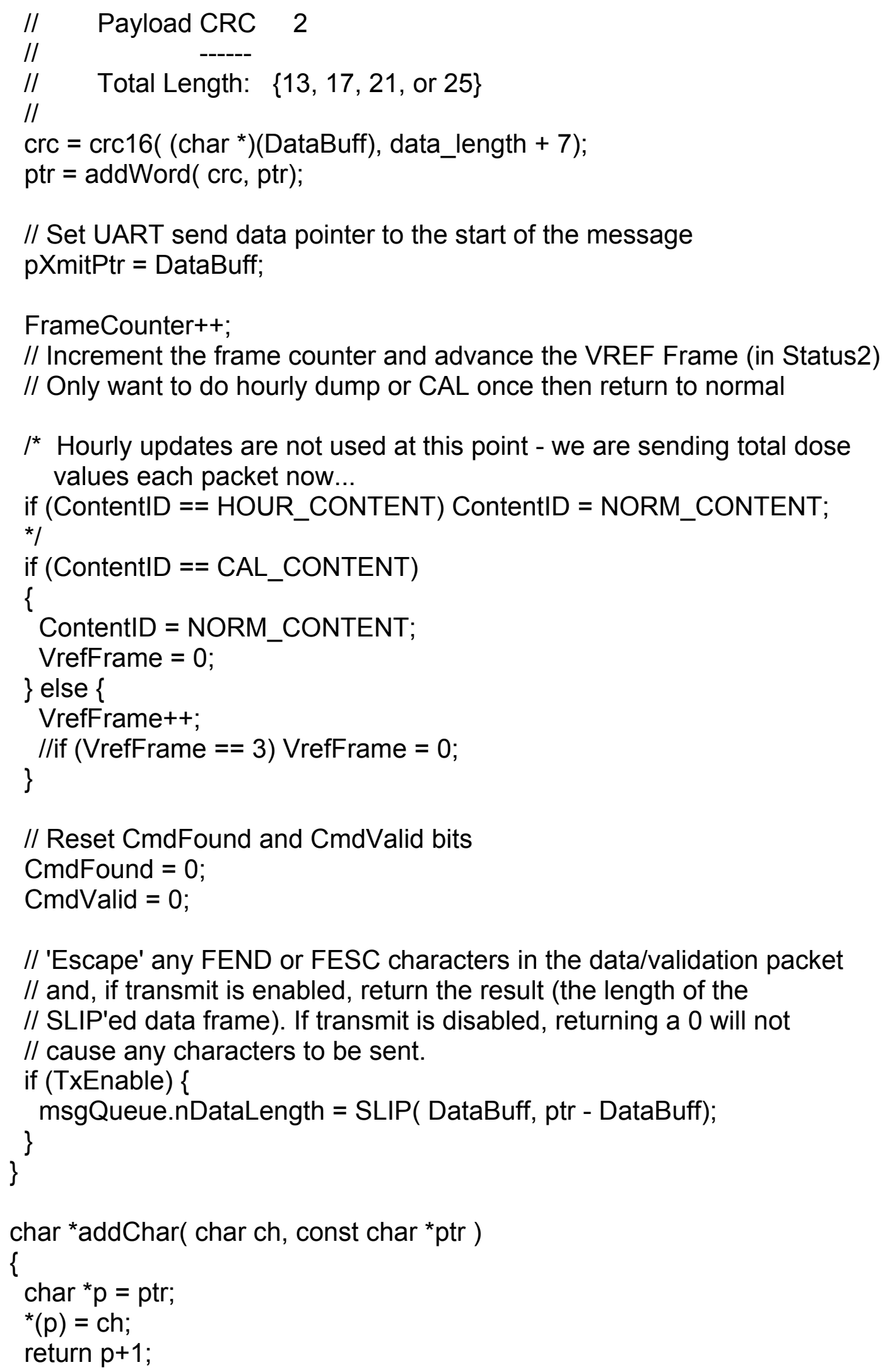

Approved for Public Release; Distribution is unlimited 


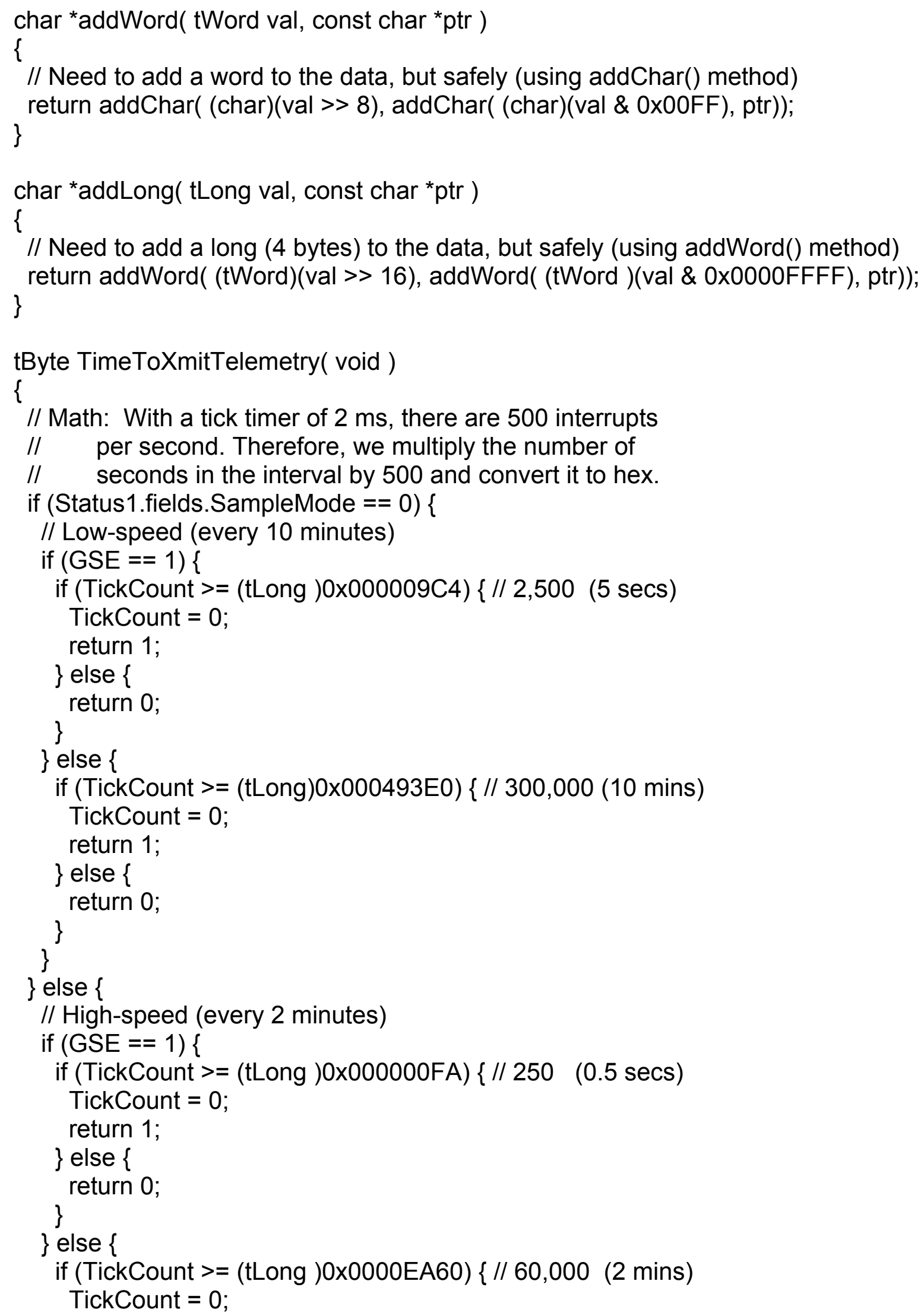

Approved for Public Release; Distribution is unlimited 


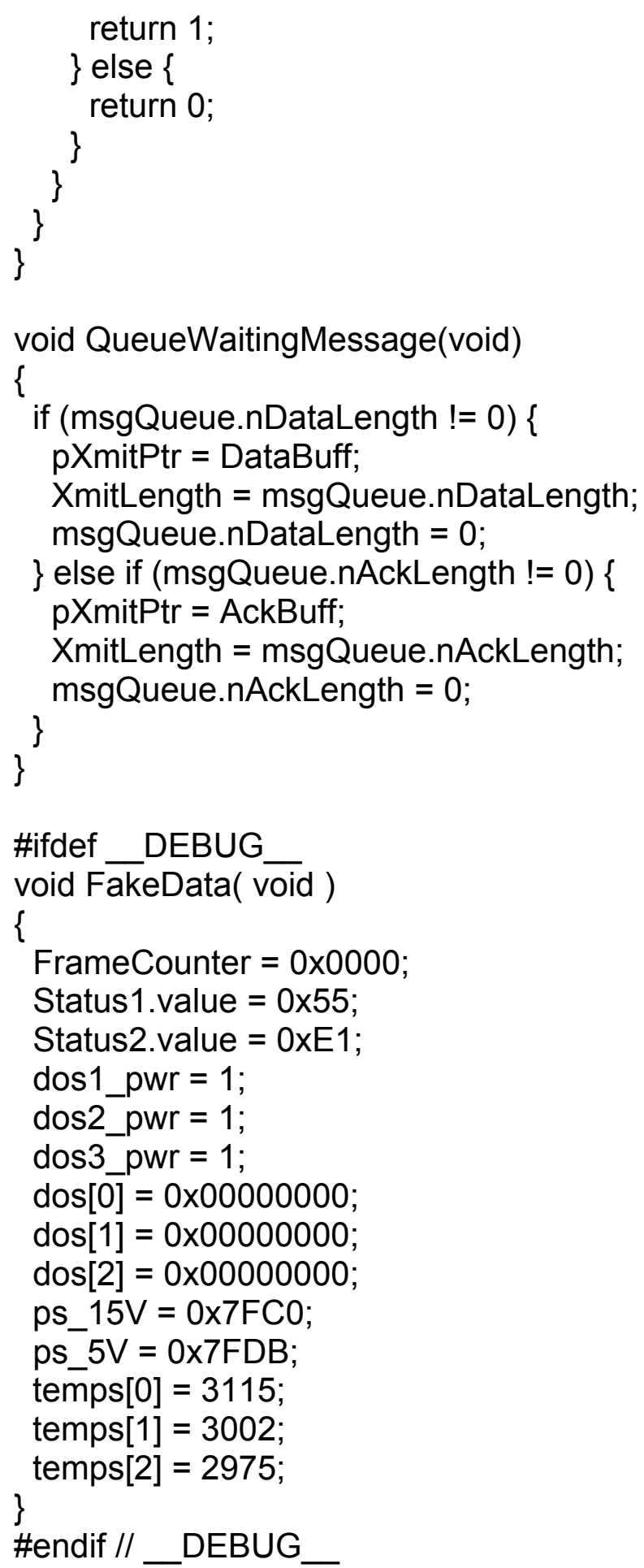


SIO.C File Reference

\#include "main.h"

Macros

- \#define TBUF_SIZE 16 $(2,4,8,16,32,64,128) * * * /$

- \#define RBUF_SIZE 16 $(2,4,8,16,32,64,128){ }^{* * *}$

$I^{* \star *}$ Must be one of these powers of 2

$/^{* * *}$ Must be one of these powers of 2

- \#define TBUF_SPACE idata $\quad I^{* * *}$ Memory space where the transmit buffer resides $* * * /$

- \#define RBUF_SPACE idata $\quad{ }^{* * *}$ Memory space where the receive buffer resides ${ }^{* *} /$

- \#define CTRL_SPACE data $\left.\right|^{* * *}$ Memory space for the buffer indexes ${ }^{* * * /}$

\section{Macro Definition Documentation}

\#define CTRL_SPACE data /*** Memory space for the buffer indexes ***/

Definition at line 26 of file SIO.C.

\#define RBUF_SIZE $16 \quad$ /*** Must be one of these powers of 2 $(2,4,8,16,32,64,128) * * * /$

Definition at line 21 of file SIO.C.

\#define RBUF_SPACE idata $\quad$ /*** Memory space where the receive buffer resides $* * * /$

Definition at line 24 of file SIO.C.

\#define TBUF_SIZE $16 \quad$ /*** Must be one of these powers of 2 $(2,4,8,16,32,64,128) * * *$

Definition at line 20 of file SIO.C. \#define TBUF_SPACE idata $\quad{ }^{* * *}$ Memory space where the transmit buffer resides $* * * /$

Definition at line 23 of file SIO.C. 
$I^{*}$

SIO.C: Serial Communication Routines.

Copyright 1995-2002 KEIL Software, Inc.

\#include "main.h"

/*-

Notes:

The length of the receive and transmit buffers must be a power of 2 .

Each buffer has a next_in and a next_out index.

If next_in = next_out, the buffer is empty.

(next_in - next_out) \% buffer_size = the number of characters in the buffer.

\begin{tabular}{|c|c|}
\hline $\begin{array}{l}\text { \#define TBUF_SIZE } 16 \\
(2,4,8,16,32,64,128) * * *\end{array}$ & $/^{* * *}$ Must be one of these powers of 2 \\
\hline $\begin{array}{l}\text { \#define RBUF_SIZE } 16 \\
(2,4,8,16,32,64,128)^{* * *} /\end{array}$ & ${ }^{* * *}$ Must be \\
\hline
\end{tabular}

\#define TBUF_SPACE idata $\quad \quad^{* * *}$ Memory space where the transmit buffer resides $* * *$

\#define RBUF_SPACE idata $\quad /^{* * *}$ Memory space where the receive buffer resides $* * *$

\#define CTRL_SPACE data $\left.\right|^{* * *}$ Memory space for the buffer indexes ${ }^{* * * /}$

\#define INTERRUPT_UART $\left.4 \quad\right|^{* * *}$ Interrupt level for Serial Port

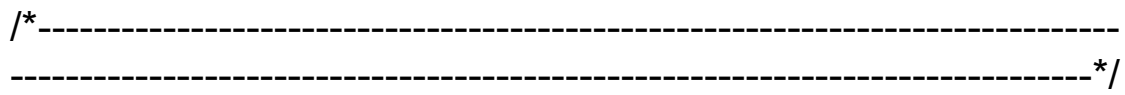

\#if TBUF_SIZE $<2$

\#error TBUF_SIZE is too small. It must be larger than 1.

\#elif TBUF_SIZE > 128

\#error TBUF_SIZE is too large. It must be smaller than 129.

\#elif ((TBUF_SIZE \& (TBUF_SIZE-1)) != 0)

\#error TBUF_SIZE must be a power of 2 .

\#endif

\#if RBUF_SIZE $<2$

\#error RBUF_SIZE is too small. It must be larger than 1.

\#elif RBUF_SIZE > 128

Approved for Public Release; Distribution is unlimited 
\#error RBUF_SIZE is too large. It must be smaller than 129.

\#elif ((RBUF_SIZE \& (RBUF_SIZE-1)) != 0)

\#error RBUF_SIZE must be a power of 2 .

\#endif

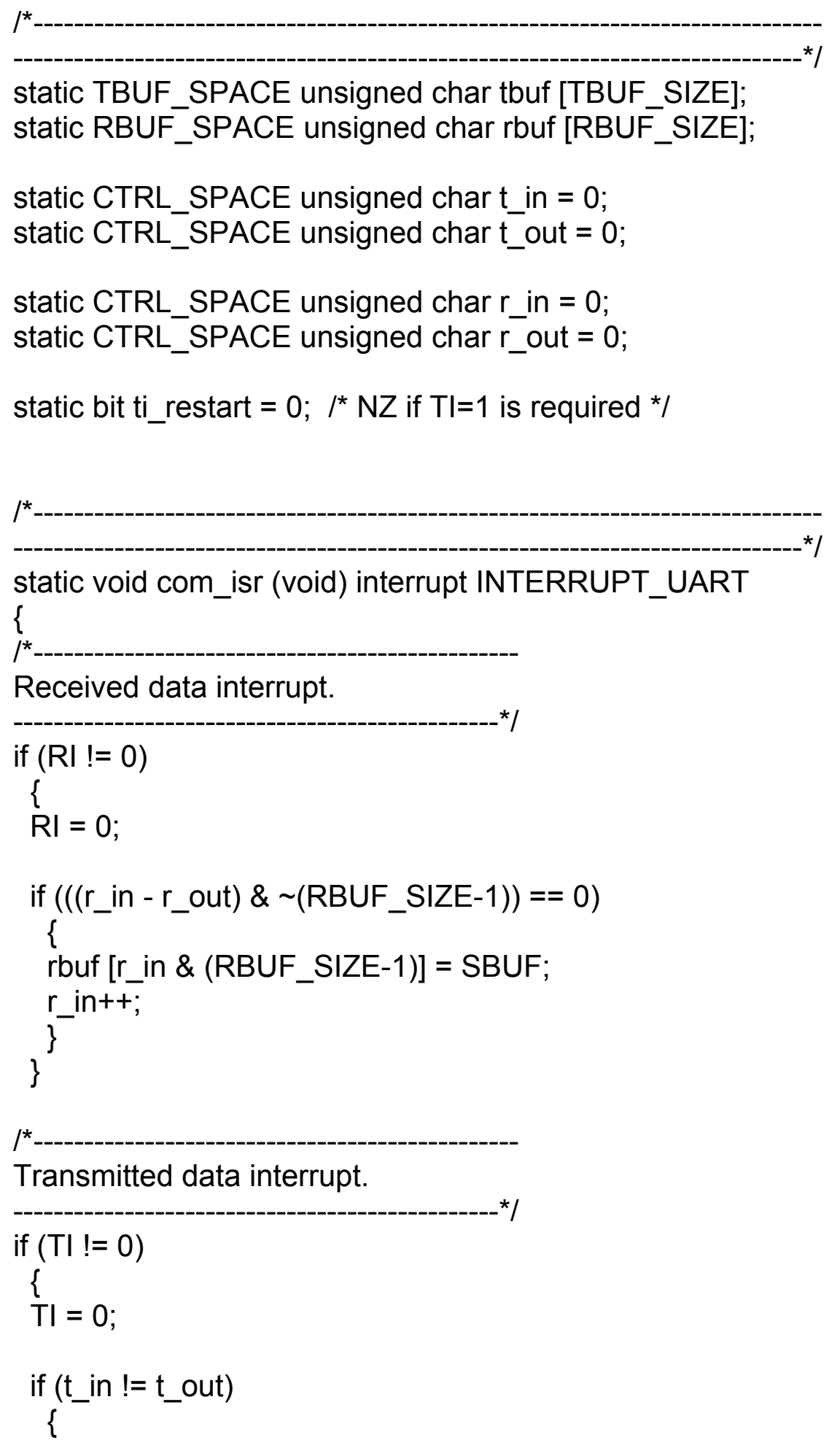

Approved for Public Release; Distribution is unlimited 


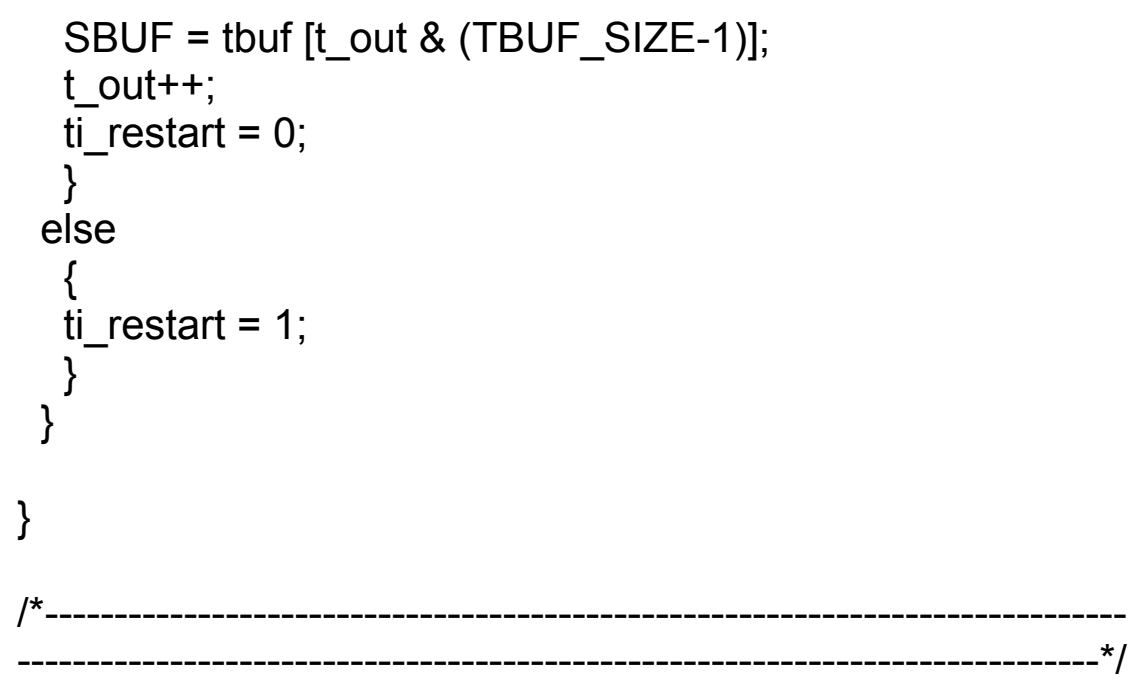

\#pragma disable

void com_initialize (void)

\{

/*

Setup TIMER1 to generate the proper baud rate.

com_baudrate (1200);

// Was 1200

$1 *$

Clear com buffer indexes.

$\mathrm{t}$ in $=0$

t_out $=0$;

$r$ in $=0$;

r_out $=0$;

$I^{*}$

Setup serial port registers.

----o-

$\mathrm{SM0}=0 ; \mathrm{SM} 1=1 ; \quad{ }^{*}$ serial port MODE $1 * /$

$\mathrm{SM} 2=0$;

$\operatorname{REN}=1 ; \quad \quad{ }^{*}$ enable serial receiver *

$\mathrm{RI}=0 ; \quad \quad \quad{ }^{*}$ clear receiver interrupt *

$\mathrm{TI}=0 ; \quad \quad I^{*}$ clear transmit interrupt *

ti_restart $=1$;

$\begin{array}{ll}\mathrm{ES}=1 ; & l^{*} \text { enable serial interrupts } * \\ \mathrm{PS}=0 ; & l^{*} \text { set serial interrupts to low priority * }\end{array}$

\}

Approved for Public Release; Distribution is unlimited 


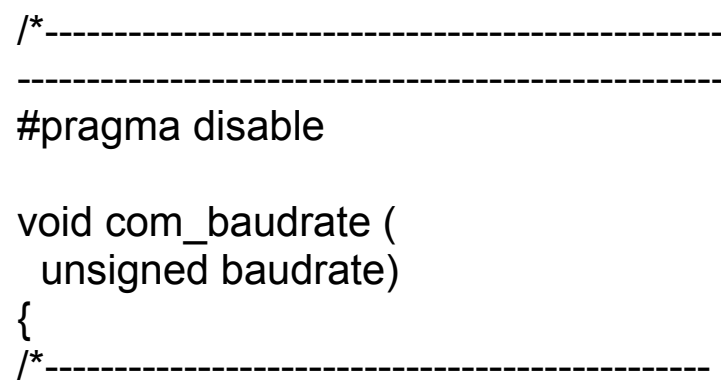

Clear transmit interrupt and buffer.

TI $=0 ;$
$\mathrm{t}$ in $=0 ; \quad{ }^{*}$ clear transmit interrupt $* /$
$\mathrm{t}$-out $=0 ;$

Set timer 1 up as a baud rate generator.

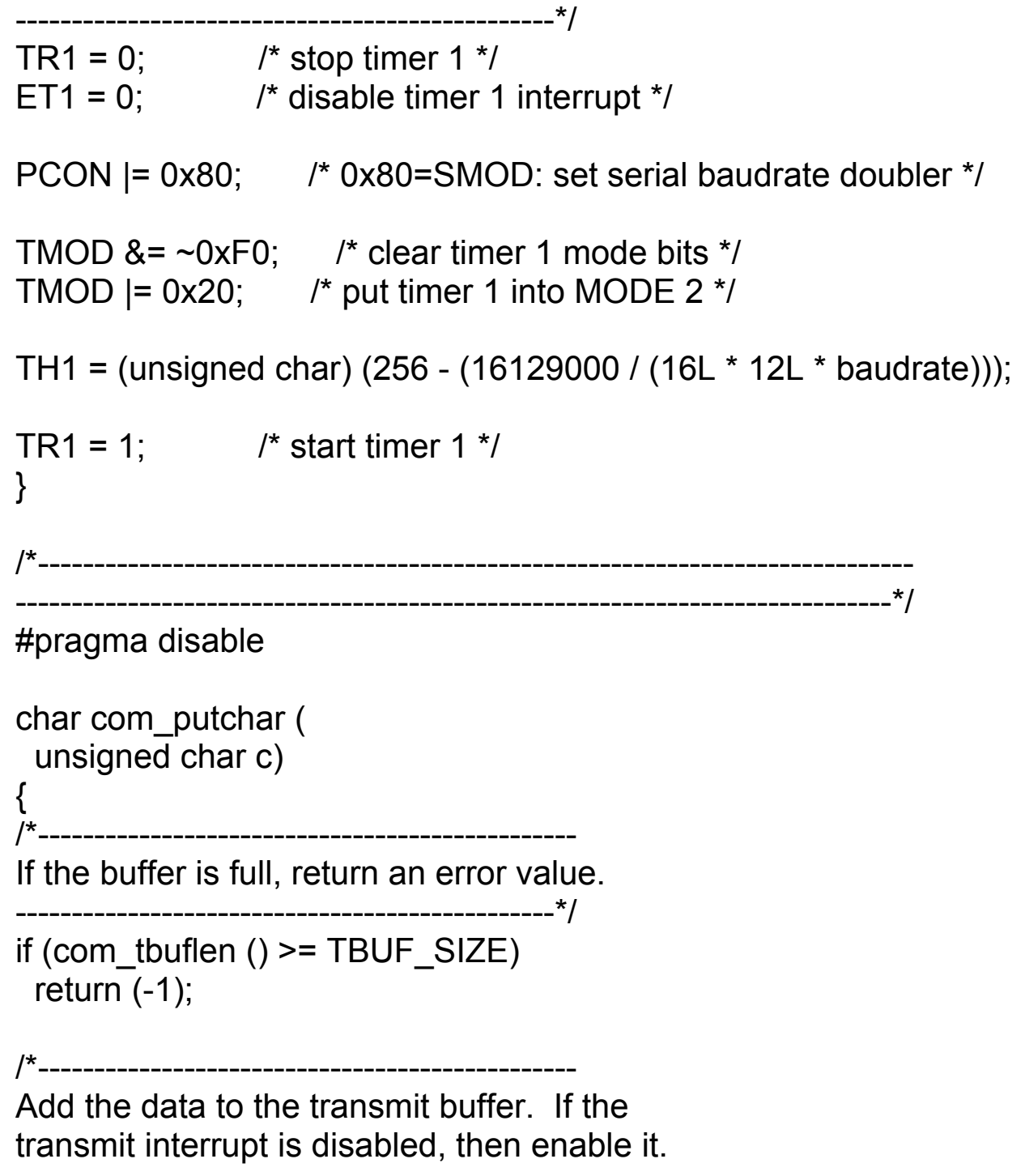

If the buffer is full, return an error value.

--o (com tbuften ()> TBUF SIZE)

if (com_tbuflen () >= TBUF_SIZE) return $(-1)$;

$I^{*}$

Add the data to the transmit buffer. If the transmit interrupt is disabled, then enable it.

Approved for Public Release; Distribution is unlimited 


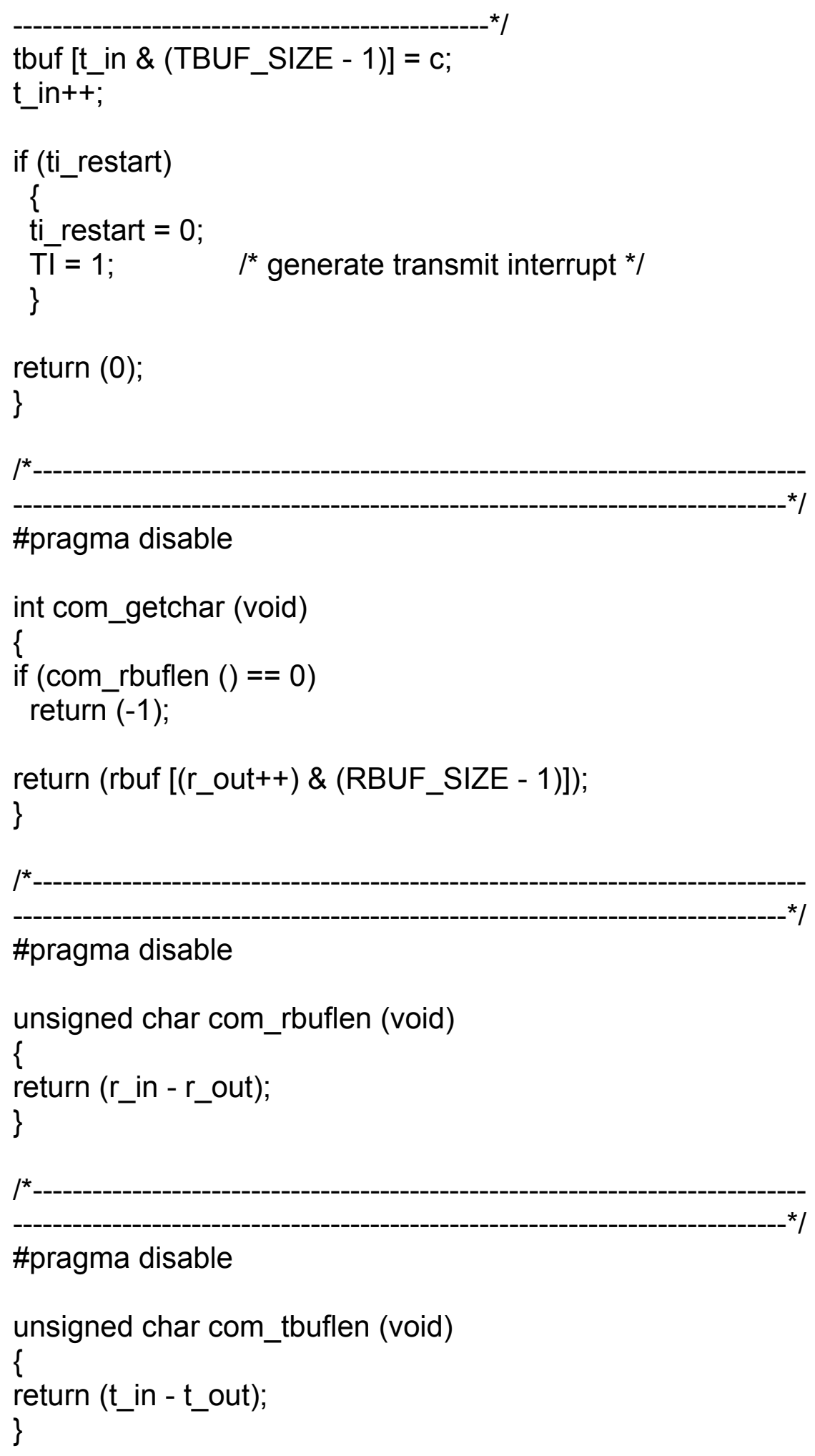




\title{
List of Abbreviations, Symbols, and Acronyms
}

\author{
AFRL - Air Force Research Laboratory \\ COSMIAC - Configurable Space Microsystems Innovations and Applications Center \\ EEPROM - Electrically Erasable Programmable Read-Only Memory \\ LEO - Low Earth Orbit \\ MIT - Massachusetts Institute of Technology \\ NASA - National Aeronautics and Space Administration \\ RHAS - Radiation Hazard Assessment System \\ SPA - Space Plug-and-play Architecture \\ SDM - Satellite Data Module \\ SSM - Satellite System Module \\ UNM - University of New Mexico \\ UNP - University Nanosat Program
}




\section{DISTRIBUTION LIST}

DTIC/OCP

8725 John J. Kingman Rd, Suite 0944

Ft Belvoir, VA 22060-6218

$1 \mathrm{cy}$

AFRL/RVIL

Kirtland AFB, NM 87117-5776

2 cys

Official Record Copy

AFRL/RVSE/Keith Avery

$1 \mathrm{cy}$

Approved for Public Release; Distribution is unlimited 